\title{
NESTA: A Fast and Accurate First-Order Method for Sparse Recovery*
}

\author{
Stephen Becker ${ }^{\dagger}$, Jérôme Bobin ${ }^{\dagger}$, and Emmanuel J. Candès ${ }^{\ddagger}$
}

\begin{abstract}
Accurate signal recovery or image reconstruction from indirect and possibly undersampled data is a topic of considerable interest; for example, the literature in the recent field of compressed sensing is already quite immense. This paper applies a smoothing technique and an accelerated first-order algorithm, both from Nesterov [Math. Program. Ser. A, 103 (2005), pp. 127-152], and demonstrates that this approach is ideally suited for solving large-scale compressed sensing reconstruction problems as (1) it is computationally efficient, (2) it is accurate and returns solutions with several correct digits, (3) it is flexible and amenable to many kinds of reconstruction problems, and (4) it is robust in the sense that its excellent performance across a wide range of problems does not depend on the fine tuning of several parameters. Comprehensive numerical experiments on realistic signals exhibiting a large dynamic range show that this algorithm compares favorably with recently proposed stateof-the-art methods. We also apply the algorithm to solve other problems for which there are fewer alternatives, such as total-variation minimization and convex programs seeking to minimize the $\ell_{1}$ norm of $W x$ under constraints, in which $W$ is not diagonal. The code is available online as a free package in the MATLAB language.
\end{abstract}

Key words. Nesterov's method, smooth approximations of nonsmooth functions, $\ell_{1}$ minimization, duality in convex optimization, continuation methods, compressed sensing, total-variation minimization

AMS subject classifications. 90C06, 90C25, 94A08

DOI. $10.1137 / 090756855$

1. Introduction. Compressed sensing $[13,14,26]$ is a novel sampling theory, which is based on the discovery that one can exploit sparsity or compressibility when acquiring signals of general interest. In a nutshell, compressed sensing designs nonadaptive sampling techniques that condense the information in a compressible signal into a small amount of data. There are some indications that because of the significant reduction in the number of measurements needed to recover a signal accurately, engineers are changing the way they think about signal acquisition in areas including analog-to-digital conversion [23], digital optics, magnetic resonance imaging [42], seismics [39], and astronomy [8].

In compressed sensing, a signal $x^{0} \in \mathbb{R}^{n}$ is acquired by collecting data of the form

$$
b=A x^{0}+z,
$$

\footnotetext{
* Received by the editors April 23, 2009; accepted for publication (in revised form) October 5, 2010; published electronically January 6, 2011. This work was partially supported by ONR grants N00014-09-1-0469 and N0001408-1-0749, by DARPA grant FA8650-08-C-7853, and by the 2006 Waterman Award from the NSF.

http://www.siam.org/journals/siims/4-1/75685.html

${ }^{\dagger}$ Applied and Computational Mathematics, Caltech, Pasadena, CA 91125 (srbecker@acm.caltech.edu, bobin@ acm.caltech.edu).

¥Applied and Computational Mathematics, Caltech, Pasadena, CA 91125 (emmanuel@acm.caltech.edu). Current address: Department of Statistics, Stanford University, 390 Serra Mall, Sequoia Hall, Stanford, CA 94305 (candes@ stanford.edu).
} 
where $x^{0}$ is the signal of interest (or its coefficient sequence in a representation where it is assumed to be fairly sparse), $A$ is a known $m \times n$ "sampling" matrix, and $z$ is a noise term. In compressed sensing and elsewhere, a standard approach attempts to reconstruct $x^{0}$ by solving

$$
\begin{array}{ll}
\text { minimize } & f(x) \\
\text { subject to } & \|b-A x\|_{\ell_{2}} \leq \epsilon,
\end{array}
$$

where $\epsilon^{2}$ is an estimated upper bound on the noise power. The choice of the regularizing function $f$ depends on prior assumptions about the signal $x^{0}$ of interest: if $x^{0}$ is (approximately) sparse, an appropriate convex function is the $\ell_{1}$ norm (as advocated by the compressed sensing theory); if $x^{0}$ is a piecewise constant object, the total-variation (TV) norm provides accurate recovery results.

Solving large-scale problems such as (1.1) (think of $x^{0}$ as having millions of entries as in megapixel images) is challenging. Although we cannot review in detail the vast literature on this subject, the majority of the algorithms that have been proposed are unable to solve these problems accurately with low computational complexity. On the one hand, standard second-order methods such as interior point methods $[10,38,52]$ are accurate but problematic, for they need to solve large systems of linear equations to compute the Newton steps. On the other hand, inspired by iterative thresholding ideas [24, 32, 20], we now have available a great number of first-order methods (see [33, 9, 36, 37] and the many earlier references therein) which may be faster but not necessarily accurate. Indeed, these methods are shown to converge slowly and typically need a very large number of iterations when high accuracy is required.

We would like to focus on the demand for high accuracy since this is the main motivation of the present paper. While in some applications one may be content with one or two digits of accuracy, there are situations in which this is simply unacceptable. Imagine that the matrix $A$ models a device giving information about the signal $x^{0}$, such as an analog-to-digital converter, for example. Here, the ability to detect and recover low power signals that are barely above the noise floor, and possibly further obscured by large interferers, is critical to many applications. In mathematical terms, one could have a superposition of high power signals corresponding to components $x^{0}[i]$ of $x^{0}$ with magnitude of order 1 , and low power signals with amplitudes as far down as $100 \mathrm{~dB}$, corresponding to components with magnitude of about $10^{-5}$. In this regime of high dynamic range, very high accuracy is required. In the example above, one would need at least five digits of precision as, otherwise, the low power signals would go undetected.

Another motivation is solving (1.1) accurately when the signal $x^{0}$ is not exactly sparse but rather approximately sparse, as in the case of real-world compressible signals. Since exactly sparse signals are rarely found in applications - while compressible signals are ubiquitous - it is important to have an accurate first-order method to handle realistic signals.

1.1. Contributions. A few years ago, Nesterov [47] published a seminal paper which couples smoothing techniques with an improved gradient method to derive first-order methods ${ }^{1}$ which achieve a convergence rate that he had proved to be optimal [45] two decades earlier.

\footnotetext{
${ }^{1}$ By "first-order," we mean methods which assume only that the local gradient information is available from a black-box oracle, but that once queried, the information persists; this is in contrast to a "pure" first-order method that uses only information about the current gradient.
}

Copyright (c) by SIAM. Unauthorized reproduction of this article is prohibited. 
As a consequence of this breakthrough, a few recent works have followed up with improved techniques in signal or image processing: in [4] a fast iterative thresholding algorithm was introduced to solve a class of composite functions that include $\left(\mathrm{QP}_{\lambda}\right)$, and $[21,56,2]$ focused on TV minimization problems. In truth, these novel algorithms demonstrate great promise; they are fast, accurate, and robust in the sense that their performance does not depend on the fine tuning of various controlling parameters.

This paper also focuses on applying some of Nesterov's work [47] discussed above, and proposes an algorithm - or, better said, a class of algorithms - for solving recovery problems from incomplete measurements. We refer to this algorithm as NESTA - short for Nesterov's algorithm - to acknowledge the fact that it is based on his method. The main purpose and the contribution of this paper consist of showing that NESTA obeys the following desirable properties:

1. Speed. We will emphasize the case where $A^{*} A$ is an orthogonal projector. Though a special case, it is widespread in compressed sensing applications. In this case, NESTA is an iterative algorithm, where each iteration is decomposed into three steps, each involving only a few matrix-vector operations. This, together with the accelerated convergence rate of Nesterov's algorithm [47, 4], makes NESTA a method of choice for solving large-scale problems. Furthermore, NESTA's convergence is driven mainly by a heuristically chosen single smoothing parameter $\mu$ introduced in section 2.1. One can use continuation techniques $[36,37]$ to dynamically update this parameter to substantially accelerate this algorithm.

2. Accuracy. NESTA depends on a few parameters that can be set in a very natural fashion. In fact, there is a clear relationship between the value of these parameters and the desired accuracy. Furthermore, our numerical experiments demonstrate that NESTA can find up to the first 4 or 5 significant digits of the optimal solution to (1.1), where $f(x)$ is the $\ell_{1}$ norm or the TV norm of $x$, in a few hundred iterations. This makes NESTA amenable to solving recovery problems involving signals of very large sizes that also exhibit a great dynamic range.

3. Flexibility. NESTA can be adapted to solve many problems beyond $\ell_{1}$ minimization with the same efficiency, such as TV minimization problems. In this paper, we will also discuss applications in which $f$ in (1.1) is given by $f(x)=\|W x\|_{\ell_{1}}$, where one may think of $W$ as a short-time Fourier transform also known as the Gabor transform, a curvelet transform, an undecimated wavelet transform, or a combination of these, or a general arbitrary dictionary of waveforms (note that this class of recovery problems also includes weighted $\ell_{1}$ methods [16]). This is particularly interesting because recent work [31] suggests the potential advantage of this analysis-based approach over the classical basis pursuit in solving important inverse problems [31].

A consequence of these properties is that NESTA, and, more generally, Nesterov's method, may be of interest to researchers working in the broad area of signal recovery from indirect and/or undersampled data.

Another contribution of this paper is that it also features a fairly wide range of numerical experiments comparing various methods against problems involving realistic and challenging data. By challenging, we mean problems of very large scale where the unknown solution exhibits a large dynamic range, that is, problems for which classical second-order methods are too slow, and for which standard first-order methods do not provide sufficient accuracy. More

Copyright (c) by SIAM. Unauthorized reproduction of this article is prohibited. 
specifically, section 5 presents a comprehensive series of numerical experiments which illustrate the behavior of several state-of-the-art methods including interior point methods [38], projected gradient techniques $[36,55,33]$, and fixed point continuation and iterative thresholding algorithms $[36,60,4]$. Most of these methods either have been perfected after several years of research $[38,33]$ or are very recent state-of-the-art methods building on insight from the past few years. For example, the fixed point continuation method with active set [37], which represents a notable improvement over existing ideas, was released while we were working on this paper (and [1] was released while we were revising the paper).

1.2. Organization of the paper and notation. As emphasized earlier, NESTA is based on Nesterov's ideas, and section 2.1 gives a brief but essential description of Nesterov's algorithmic framework. The proposed algorithm is introduced in section 3. Inspired by continuationlike schemes, an accelerated version of NESTA is described in section 3.6. We report on extensive and comparative numerical experiments in section 5. Section 6 covers extensions of NESTA to minimize the $\ell_{1}$ norm of $W x$ under data constraints (section 6.1) and includes realistic simulations in the field of radar pulse detection and estimation. Section 6.3 extends NESTA to solve TV problems and presents numerical experiments which also demonstrate its remarkable efficiency there as well. Finally, we conclude with section 7, discussing further extensions which would address an even wider range of linear inverse problems.

Notation. Before we begin, it is best to provide a brief summary of the notation used throughout the paper. As usual, vectors are written in small letters and matrices in capital letters. The $i$ th entry of a vector $x$ is denoted $x[i]$, and the $(i, j)$ th entry of the matrix $A$ is $A[i, j]$.

It is convenient to introduce some common optimization problems that will be discussed throughout. Solving sparse reconstruction problems can be approached via several different equivalent formulations. In this paper, we particularly emphasize the quadratically constrained $\ell_{1}$ minimization problem

$$
\begin{array}{lll}
\left(\mathrm{BP}_{\epsilon}\right) & \text { minimize } & \|x\|_{\ell_{1}} \\
& \text { subject to } & \|b-A x\|_{\ell_{2}} \leq \epsilon,
\end{array}
$$

where $\epsilon$ quantifies the uncertainty about the measurements $b$ as in the situation where the measurements are noisy. This formulation is often preferred because a reasonable estimate of $\epsilon$ may be known. A second frequently discussed approach considers solving this problem in Lagrangian form, i.e.,

$$
\left(\mathrm{QP}_{\lambda}\right) \quad \text { minimize } \quad \lambda\|x\|_{\ell_{1}}+\frac{1}{2}\|b-A x\|_{\ell_{2}}^{2},
$$

and is also known as the basis pursuit denoising problem [18]. This problem is popular in signal and image processing [24,32]; it is often interpreted as a maximum a posteriori estimate in a Bayesian setting or, simply, as a regularized least-squares problem. In statistics, the same problem is better known as the LASSO [53]:

$$
\begin{aligned}
& \left(\mathrm{LS}_{\tau}\right) \quad \text { minimize } \quad\|b-A x\|_{\ell_{2}} \\
& \text { subject to } \quad\|x\|_{\ell_{1}} \leq \tau \text {. }
\end{aligned}
$$

Copyright (c) by SIAM. Unauthorized reproduction of this article is prohibited. 
Standard optimization theory [51] asserts that these three problems are, of course, equivalent, provided that $\epsilon, \lambda, \tau$ obey some special relationships. With the exception of the case where the matrix $A$ is orthogonal, this functional dependence is hard to compute [55]. Because it is usually more natural to determine an appropriate $\epsilon$ rather than an appropriate $\lambda$ or $\tau$, the fact that NESTA solves $\left(\mathrm{BP}_{\epsilon}\right)$ is a significant advantage. Further, note that theoretical equivalence of course does not mean that all three problems are just as easy (or just as hard) to solve. For instance, the constrained problem $\left(\mathrm{BP}_{\epsilon}\right)$ may be harder to solve than $\left(\mathrm{QP}_{\lambda}\right)$. Hence, the fact that NESTA turns out to be competitive with algorithms that solve only $\left(\mathrm{QP}_{\lambda}\right)$ is quite remarkable.

\section{NESTA.}

2.1. Nesterov's method to minimize smooth convex functions. In [46, 45], Nesterov introduces a subtle algorithm to minimize any smooth convex function $f$ on the convex set $\mathcal{Q}_{p}$,

$$
\min _{x \in \mathcal{Q}_{p}} f(x) .
$$

We will refer to $\mathcal{Q}_{p}$ as the primal feasible set. The function $f$ is assumed to be differentiable, and its gradient $\nabla f(x)$ is Lipschitz and obeys

$$
\forall x, y \in \operatorname{dom}(f), \quad\|\nabla f(x)-\nabla f(y)\|_{\ell_{2}} \leq L\|x-y\|_{\ell_{2}},
$$

where $\operatorname{dom}(f) \equiv \mathbb{R}^{n}$ and $L$ is an upper bound on the Lipschitz constant. With these assumptions, Nesterov's algorithm minimizes $f$ over $\mathcal{Q}_{p}$ by iteratively estimating three sequences $\left\{x_{k}\right\},\left\{y_{k}\right\}$, and $\left\{z_{k}\right\}$ while smoothing the feasible set $\mathcal{Q}_{p}$. The algorithm depends on two scalar sequences $\left\{\alpha_{k}\right\}$ and $\left\{\tau_{k}\right\}$ discussed below and takes the following form (this specific form is based on [47]).

Initialize $x_{0}$. For $k \geq 0$,

1. Compute $\nabla f\left(x_{k}\right)$.

2. Compute $y_{k}$ :

$$
y_{k}=\underset{x \in \mathcal{Q}_{p}}{\operatorname{argmin}} \frac{L}{2}\left\|x-x_{k}\right\|_{\ell_{2}}^{2}+\left\langle\nabla f\left(x_{k}\right), x-x_{k}\right\rangle .
$$

3. Compute $z_{k}$ :

$$
z_{k}=\underset{x \in \mathcal{Q}_{p}}{\operatorname{argmin}} L p_{p}(x)+\sum_{i=0}^{k} \alpha_{i}\left\langle\nabla f\left(x_{i}\right), x-x_{i}\right\rangle .
$$

4. Update $x_{k}$ :

$$
x_{k+1}=\tau_{k} z_{k}+\left(1-\tau_{k}\right) y_{k} .
$$

Stop when a given criterion is valid. 
At step $k, y_{k}$ is the current guess of the optimal solution. If we performed only the second step of the algorithm with $y_{k-1}$ instead of $x_{k}$, we would obtain a standard first-order technique with convergence rate $\mathcal{O}(1 / k)$. This second step can be seen as minimizing an approximate Taylor expansion of $f$ about $x_{k}$ by taking an upper bound on the Hessian.

The novelty is that the sequence $z_{k}$ "keeps in mind" the previous iterations since step 3 involves a weighted sum of already computed gradients. ${ }^{2}$ Another aspect of this step is thatborrowing ideas from smoothing techniques in optimization - it makes use of a prox-function $p_{p}(x)$ for the primal feasible set $\mathcal{Q}_{p}$. This function is strongly convex with parameter $\sigma_{p}$. Without loss of generality, we will set $\sigma_{p}=1$. Assuming that $p_{p}(x)$ vanishes at the proxcenter $x_{p}^{c}=\operatorname{argmin}_{x} p_{p}(x)$, this gives

$$
p_{p}(x) \geq \frac{1}{2}\left\|x-x_{p}^{c}\right\|_{\ell_{2}}^{2} .
$$

The prox-function is usually chosen so that $x_{p}^{c} \in \mathcal{Q}_{p}$, thus discouraging $z_{k}$ from moving too far away from the center $x_{p}^{c}$.

The point $x_{k}$, at which the gradient of $f$ is evaluated, is a weighted average between $z_{k}$ and $y_{k}$. This is motivated by a theoretical analysis [47, 54], which shows that if ${ }^{3} \alpha_{k}=1 / 2(k+1)$ and $\tau_{k}=2 /(k+3)$, then the sequence $\left\{f\left(y_{k}\right)\right\}$ ( $y_{k}$ is feasible) converges to $f\left(x^{\star}\right)$, where $x^{\star}=\operatorname{argmin}_{x \in \mathcal{Q}_{p}} f(x)$, with the convergence rate (see [47, Theorem 2])

$$
f\left(y_{k}\right)-f\left(x^{\star}\right) \leq \frac{4 L p_{p}\left(x^{\star}\right)}{(k+1)^{2}} .
$$

This decay is far better than what is achievable via standard gradient-based optimization techniques since we have an approximation scaling like $L / k^{2}$ instead of $L / k$.

3. Application to compressed sensing. We now apply Nesterov's algorithm to solve compressed sensing recovery problems, and we refer to this extension as NESTA. For now, we shall be concerned with solving the quadratically constrained $\ell_{1}$ minimization problem (1.2).

3.1. NESTA. We wish to solve (1.2), i.e., minimize $\|x\|_{\ell_{1}}$ subject to $\|b-A x\|_{\ell_{2}} \leq \epsilon$, where $A \in \mathbb{R}^{m \times n}$ is singular $(m<n)$.

In this paper, we assume that $A^{*} A$ is an orthogonal projector; i.e., the rows of $A$ are orthonormal. This is often the case in compressed sensing applications where it is common to take $A$ as a submatrix of a unitary transformation which admits a fast algorithm for matrixvector products; special instances include the discrete Fourier transform (DFT), the discrete cosine transform (DCT), the Hadamard transform, and the noiselet transform. Basically, collecting incomplete structured orthogonal measurements is the prime method for efficient data acquisition in compressed sensing. Section 7 discusses different approaches for relaxing this assumption.

\footnotetext{
${ }^{2}$ Using the sequence $\left\{\nabla f\left(x_{i}\right)\right\}_{1 \leq i \leq k}$ to update the current estimate $x_{k}$ is not new. For instance, in algorithms such as SESOP [30], the search direction is optimally evaluated at each iteration in the subspace spanned by previous gradients; this requires storing a set of previous values, which may not be practical for large-scale problems.

${ }^{3}$ Other choices for $\alpha_{k}$ and $\tau_{k}$ are possible [47].
} 
From nonsmooth to smooth functions. The algorithm described in the previous paragraph has been introduced to minimize a smooth function $f$; it cannot be applied directly to solve $\left(\mathrm{BP}_{\epsilon}\right)$, as the function $f(x)=\|x\|_{\ell_{1}}$ is not smooth. In an innovative paper [47], Nesterov recently extended this framework to deal with a certain class of nonsmooth convex functions. Suppose that the function $f$ to be minimized has the form

$$
f(x)=\max _{u \in \mathcal{Q}_{d}}\langle u, W x\rangle,
$$

where $x \in \mathbb{R}^{n}, u \in \mathbb{R}^{p}$, and $W \in \mathbb{R}^{p \times n}$. $W$ is assumed to have full column rank. We will refer to $\mathcal{Q}_{d}$ as the dual feasible set and assume it is convex. In [47], Nesterov proposed substituting $f$ by the smooth approximation

$$
f_{\mu}(x)=\max _{u \in \mathcal{Q}_{d}}\langle u, W x\rangle-\mu p_{d}(u),
$$

where $p_{d}(u)$ is a prox-function for $\mathcal{Q}_{d}$; that is, $p_{d}(u)$ is continuous and strongly convex on $\mathcal{Q}_{d}$, with convexity parameter $\sigma_{d}$ which we will take to be 1 . Nesterov proved that $f_{\mu}$ is continuously differentiable and that its gradient obeys

$$
\nabla f_{\mu}(x)=W^{*} u_{\mu}(x)
$$

where $u_{\mu}(x)$ is the optimal solution of (3.2). Furthermore, $\nabla f_{\mu}$ is shown to be Lipschitz with constant

$$
L=\frac{1}{\mu \sigma_{d}}\|W\|^{2}
$$

$(\|W\|$ is the operator norm of $W)$. It is now possible to apply Nesterov's accelerated method [47] to the smoothed function $f_{\mu}$. This produces a feasible sequence of points that will weakly converge to the minimizer of the smoothed problem at rate $\mathcal{O}\left(k^{-2}\right)$, and, empirically, the minimizers of the smoothed problem and the unsmoothed problem can be made arbitrarily close by taking $\mu \rightarrow 0$.

The particular case $f(x)=\|x\|_{\ell_{1}}$. In this setting, the $\ell_{1}$ norm can be formulated as in (3.1) since

$$
\|x\|_{\ell_{1}}=\max _{u \in \mathcal{Q}_{d}}\langle u, x\rangle,
$$

where the dual feasible set $\mathcal{Q}_{d}$ is the $\ell_{\infty}$ ball $\mathcal{Q}_{d}=\left\{u:\|u\|_{\infty} \leq 1\right\}$. Therefore, a natural smooth approximation to the $\ell_{1}$ norm is

$$
f_{\mu}(x)=\max _{u \in \mathcal{Q}_{d}}\langle u, x\rangle-\mu p_{d}(u),
$$

where $p_{d}(u)$ is our dual prox-function. For $p_{d}(u)$, we would like a strongly convex function, which is known analytically and takes its minimum value (equal to zero) at some $u_{d}^{c} \in \mathcal{Q}_{d}$. It is also usual to have $p_{d}(u)$ separable. Taking these criteria into account, a convenient choice is $p_{d}(u)=\frac{1}{2}\|u\|_{\ell_{2}}^{2}$, whose strong convexity parameter is equal to 1 . With this prox-function,

Copyright ( $\odot$ by SIAM. Unauthorized reproduction of this article is prohibited. 
$f_{\mu}$ is the well-known Huber function ${ }^{4}$ and $\nabla f_{\mu}$ is Lipschitz with constant $1 / \mu$. In particular, $\nabla f_{\mu}(x)$ is given by

$$
\nabla f_{\mu}(x)[i]= \begin{cases}\mu^{-1} x[i] & \text { if }|x[i]|<\mu, \\ \operatorname{sgn}(x[i]) & \text { otherwise. }\end{cases}
$$

As proposed in [47], Nesterov's algorithm can then be applied to solve

$$
\min _{x \in \mathcal{Q}_{p}} f_{\mu}(x)
$$

where $\mathcal{Q}_{p}=\left\{x:\|b-A x\|_{\ell_{2}} \leq \epsilon\right\}$. Let us note that this problem is an approximation to $\left(\mathrm{BP}_{\epsilon}\right)$ with $0 \leq f(x)-f_{\mu}(x) \leq n \mu / 2$ for all $x \in \mathbb{R}^{n}$; this bound is overly pessimistic if $x$ is $s$-sparse, since $f(0)=f_{\mu}(0)$, so the expected approximation error is closer to $s \mu / 2$ for these signals.

The only remaining details of the algorithm are about the auxiliary updates of $y_{k}$ and $z_{k}$ in steps 2 and 3 . In the next section, we show that these steps are computationally very cheap when $A^{*} A$ is assumed to be an orthogonal projector. ${ }^{5}$

3.2. Updating $y_{k}$. To compute $y_{k}$, we need to solve a problem of the form

$$
y_{k}=\underset{x \in \mathcal{Q}_{p}}{\operatorname{argmin}} \frac{L}{2}\|d-x\|_{\ell_{2}}^{2}+\left\langle c, x-x_{k}\right\rangle,
$$

where $x_{k}$ is from the previous iterate and $L$ is given by (3.4), $d=x_{k}$, and $c=\nabla f_{\mu}\left(x_{k}\right)$. The Lagrangian for this problem is

$$
\mathcal{L}(x, \lambda)=\frac{L}{2}\|d-x\|_{\ell_{2}}^{2}+\frac{\lambda}{2}\left(\|b-A x\|_{\ell_{2}}^{2}-\epsilon^{2}\right)+\left\langle c, x-x_{k}\right\rangle,
$$

and at the primal-dual solution $\left(y_{k}, \lambda_{\epsilon}\right)$, the Karush-Kuhn-Tucker (KKT) conditions [51] read as

$$
\begin{aligned}
\left\|b-A y_{k}\right\|_{\ell_{2}} & \leq \epsilon, \\
\lambda_{\epsilon} & \geq 0, \\
\lambda_{\epsilon}\left(\left\|b-A y_{k}\right\|_{\ell_{2}}^{2}-\epsilon^{2}\right) & =0, \\
L\left(y_{k}-d\right)+\lambda_{\epsilon} A^{*}\left(A y_{k}-b\right)+c & =0 .
\end{aligned}
$$

From the stationarity condition, $y_{k}$ is the solution to the linear system

$$
\left(I+\frac{\lambda_{\epsilon}}{L} A^{*} A\right) y_{k}=\frac{\lambda_{\epsilon}}{L} A^{*} b+d-\frac{1}{L} c .
$$

As discussed earlier, our assumption is that $A^{*} A$ is an orthogonal projector so that

$$
y_{k}=\left(I-\frac{\lambda_{\epsilon}}{\lambda_{\epsilon}+L} A^{*} A\right)\left(\frac{\lambda_{\epsilon}}{L} A^{*} b+d-\frac{1}{L} c\right) .
$$

\footnotetext{
${ }^{4}$ The smoothed version of the TV norm is not known to have a specific name.

${ }^{5}$ Taking $x_{0}=A^{*} b$ which is feasible and observing that $f\left(x^{\star}\right) \leq f\left(A^{*} b\right)$ for any minimizer $x^{\star}$ implies the boundedness of $x^{\star}$ and, hence, that of $p_{p}\left(x^{\star}\right)$ in (2.3). This follows from $f(x) \leq\|x\|_{\ell_{1}} \leq f(x)+\mu n / 2$ valid for all $x$, giving $\left\|x^{\star}\right\|_{\ell_{1}} \leq f\left(A^{*} b\right)+\mu n / 2$.
} 
In this case, computing $y_{k}$ is cheap since no matrix inversion is required - only a few matrixvector products are necessary. Moreover, from the KKT conditions, the value of the optimal Lagrange multiplier is obtained explicitly and equals

$$
\lambda_{\epsilon}=\max \left(0, L \epsilon^{-1}\|b-A q\|_{\ell_{2}}-L\right), \quad q=d-L^{-1} c,
$$

where $c=\nabla f_{\mu}\left(x_{k}\right)$ and $d=x_{k}$. Observe that this can be computed beforehand since it depends only on $x_{k}$ and $\nabla f_{\mu}\left(x_{k}\right)$.

3.3. Updating $z_{k}$. To compute $z_{k}$, we need to solve

$$
z_{k}=\underset{x \in \mathcal{Q}_{p}}{\operatorname{argmin}} L p_{p}(x)+\left\langle\sum_{i \leq k} \alpha_{i} \nabla f_{\mu}\left(x_{i}\right), x-x_{k}\right\rangle,
$$

where $p_{p}(x)$ is the primal prox-function. The point $z_{k}$ differs from $y_{k}$ since it is computed from a weighted cumulative gradient $\sum_{i<k} \alpha_{i} \nabla f_{\mu}\left(x_{i}\right)$, making it less prone to zig-zagging, which typically occurs when we have highly elliptical level sets. This step keeps a memory from the previous steps and forces $z_{k}$ to stay near the prox-center. A good primal prox-function is a smooth and strongly convex function that is likely to have some positive effect near the solution. In the setting of (1.1), a suitable smoothing prox-function may be

$$
p_{p}(x)=\frac{1}{2}\left\|x-x_{0}\right\|_{\ell_{2}}^{2}
$$

for some $x_{0} \in \mathbb{R}^{n}$, e.g., an initial guess of the solution. Other choices of primal feasible set $\mathcal{Q}_{p}$ may lead to other choices of prox-functions. For instance, when $\mathcal{Q}_{p}$ is the standard simplex, choosing an entropy distance for $p_{p}(x)$ is smarter and more efficient; see [47]. In this paper, the primal feasible set is quadratic, which makes the Euclidean distance a reasonable choice. What is more important, however, is that this choice allows very efficient computations of $z_{k}$, while other choices may considerably slow down each Nesterov iteration. Finally, notice that the bound on the error at iteration $k$ in $(2.3)$ is proportional to $p_{p}\left(x^{\star}\right)$; choosing $x_{0}$ wisely (a good first guess) can make $p_{p}\left(x^{\star}\right)$ small. When nothing is known about the solution, a natural choice may be $x_{0}=A^{*} b$; this idea will be developed in section 3.6.

With (3.13), the strong convexity parameter of $p_{p}(x)$ is equal to 1 , and to compute $z_{k}$ we need to solve a quadratically constrained quadratic program that has exactly the same formulation as (3.7), where $d=x_{0}$ and $c=\sum_{i<k} \alpha_{i} \nabla f_{\mu}\left(x_{i}\right)$. Updating $z_{k}$ is then just as cheap as updating $y_{k}$. Furthermore, many of the matrix-vector multiplications from the $y_{k}$ update can be reused.

3.4. Computational complexity. The computational complexity of each of NESTA's steps is clear. In large-scale problems, most of the work is in the application of $A$ and $A^{*}$. Define $\mathcal{C}_{A}$ to be the complexity of applying $A$ or $A^{*}$. The first step, namely, computing $\nabla f_{\mu}$, requires only vector operations whose complexity is $\mathcal{O}(n)$. Steps 2 and 3 require the application of $A$ or $A^{*}$ three times each (we need only compute $A^{*} b$ once). Hence, the total complexity of a single NESTA iteration is $6 \mathcal{C}_{A}+\mathcal{O}(n)$, where $\mathcal{C}_{A}$ is dominant.

The calculations above are in some sense overly pessimistic. In compressed sensing applications, it is common to choose $A$ as a submatrix of a unitary transformation $U$, which admits

Copyright (C) by SIAM. Unauthorized reproduction of this article is prohibited. 
a fast algorithm for matrix-vector products. In what follows, it might be useful to think of $A$ as a subsampled DFT. In this case, letting $R$ be the $m \times n$ matrix extracting the observed measurements, we have $A=R U$. The trick then is to compute in the $U$-domain directly. Making the change of variables $x \leftarrow U x$, our problem is

$$
\begin{array}{ll}
\text { minimize } & \hat{f}_{\mu}(x) \\
\text { subject to } & \|b-R x\|_{\ell_{2}} \leq \epsilon,
\end{array}
$$

where $\hat{f}_{\mu}=f_{\mu} \circ U^{*}$. The gradient of $\hat{f}_{\mu}$ is then

$$
\nabla \hat{f}_{\mu}(x)=U \nabla f_{\mu}\left(U^{*} x\right)
$$

With this change of variables, steps 2 and 3 do not require applying $U$ or $U^{*}$ since

$$
y_{k}=\left(I-\frac{\lambda}{\lambda+L} R^{*} R\right)\left(\frac{\lambda}{L} R^{*} b+x_{k}-\frac{1}{L} \nabla \hat{f}_{\mu}\left(x_{k}\right)\right),
$$

where $R^{*} R$ is the diagonal matrix with $0 / 1$ diagonal entries depending on whether or not a coordinate is sampled. As before, $\lambda_{\epsilon}=\max \left(0,\|b-R q\|_{\ell_{2}}-L\right)$ with $q=x_{k}-L^{-1} \nabla \hat{f}_{\mu}\left(x_{k}\right)$. The complexity of step 2 is now $\mathcal{O}(n)$, and the same applies to step 3.

Put $\mathcal{C}_{U}$ for the complexity of applying $U$ and $U^{*}$. The complexity of step 1 is now $2 \mathcal{C}_{U}$, so that this simple change of variables reduces the cost of each NESTA iteration to $2 \mathcal{C}_{U}+\mathcal{O}(n)$. For example, in the case of a subsampled DFT (or something similar), the cost of each iteration is essentially that of two FFTs. Hence, each iteration is extremely fast.

3.5. Parameter selection. NESTA involves the selection of a single smoothing parameter $\mu$ and a suitable stopping criterion. For the latter, our experience indicates that a robust and fairly natural stopping criterion is to terminate the algorithm when the relative variation of $f_{\mu}$ is small. Define $\Delta f_{\mu}$ as

$$
\Delta f_{\mu}:=\frac{\left|f_{\mu}\left(x_{k}\right)-\bar{f}_{\mu}\left(x_{k}\right)\right|}{\bar{f}_{\mu}\left(x_{k}\right)}, \quad \bar{f}_{\mu}\left(x_{k}\right):=\frac{1}{\min \{10, k\}} \sum_{l=1}^{\min \{10, k\}} f_{\mu}\left(x_{k-l}\right) .
$$

Then convergence is claimed when

$$
\Delta f_{\mu}<\delta
$$

for some $\delta>0$. In our experiments, $\delta \in\left\{10^{-5}, 10^{-6}, 10^{-7}, 10^{-8}\right\}$ depending upon the desired accuracy. These values might depend on the problem type and should be optimized accordingly. Clearly, $\delta$ should be small when $\mu$ is small, since this is the high accuracy situation; when $\mu$ is larger, $\delta$ may be taken to be larger as well.

The choice of $\mu$ is based on a trade-off between the accuracy of the smoothed approximation $f_{\mu}$ (basically, $\lim _{\mu \rightarrow 0} f_{\mu}(x)=\|x\|_{\ell_{1}}$ ) and the speed of convergence (the convergence rate is proportional to $\mu$ ). With noiseless data, $\mu$ is directly linked to the desired accuracy. To illustrate this, we have observed in [7] that when the true signal $x^{0}$ is exactly sparse and is actually the minimum solution under the equality constraints $A x^{0}=b$, the $\ell_{\infty}$ error on the nonzero entries is on the order of $\mu$. The link between $\mu$ and accuracy will be further discussed in section 4.3. 
3.6. Accelerating NESTA with continuation. Inspired by homotopy techniques which find the solution to the LASSO problem (1.4) for values of $\tau$ ranging in an interval $\left[0, \tau_{\max }\right]$, [36] and [25] independently introduced a fixed point continuation technique which solves $\ell_{1}$ penalized least-squares problems (1.3),

$$
\left(\mathrm{QP}_{\lambda}\right) \quad \operatorname{minimize} \quad \lambda\|x\|_{\ell_{1}}+\frac{1}{2}\|b-A x\|_{\ell_{2}}^{2}
$$

for values of $\lambda$ obeying $0<\lambda<\left\|A^{*} b\right\|_{\ell_{\infty}}$. The continuation solution approximately follows the path of solutions to the problem $\left(\mathrm{QP}_{\lambda}\right)$, and, hence, the solutions to (1.1) and (1.4) may be found by solving a sequence of $\ell_{1}$-penalized least-squares problems.

The point of this is that it has been noticed (see [36, 49, 28, 58]) that solving (1.3) (resp., the LASSO (1.4)) is faster when $\lambda$ is large (resp., $\tau$ is low). This observation greatly motivates the use of continuation for solving (1.3) for a fixed $\lambda_{f}$. The idea is simple: propose a sequence of problems with decreasing values of the parameter $\lambda, \lambda_{0}>\cdots>\lambda_{f}$, and use the intermediate solution as a warm start for the next problem. This technique has been used with success in $[33,55]$. Continuation has been shown to be a very successful tool for increasing the speed of convergence, particularly when dealing with large-scale problems and high dynamic range signals.

Likewise, our proposed algorithm can greatly benefit from a continuation approach. Recall that to compute $y_{k}$, we need to solve

$$
\begin{aligned}
y_{k} & =\underset{x \in \mathcal{Q}_{p}}{\operatorname{argmin}} \frac{L}{2}\left\|x-x_{k}\right\|_{\ell_{2}}^{2}+\langle c, x\rangle \\
& =\underset{x \in \mathcal{Q}_{p}}{\operatorname{argmin}}\left\|x-\left(x_{k}-L^{-1} c\right)\right\|_{\ell_{2}}^{2}
\end{aligned}
$$

for some vector $c$. Thus with $\mathcal{P}_{\mathcal{Q}_{p}}$ the projector onto $\mathcal{Q}_{p}, y_{k}=\mathcal{P}_{\mathcal{Q}_{p}}\left(x_{k}-L^{-1} c\right)$. Now two observations are in order.

1. Computing $y_{k}$ is similar to a projected gradient step as the Lipschitz constant $L^{-1}$ plays the role of the step size. Since $L$ is proportional to $\mu^{-1}$, the larger $\mu$ is, the larger the step size, and the faster the convergence. This also applies to the sequence $\left\{z_{k}\right\}$.

2. For a fixed value of $\mu$, the convergence rate of the algorithm obeys

$$
f_{\mu}\left(y_{k}\right)-f_{\mu}\left(x_{\mu}^{\star}\right) \leq \frac{2 L\left\|x_{\mu}^{\star}-x_{0}\right\|_{\ell_{2}}^{2}}{k^{2}},
$$

where $x_{\mu}^{\star}$ is the optimal solution to $\min f_{\mu}$ over $\mathcal{Q}_{p}$. On the one hand, the convergence rate is proportional to $\mu^{-1}$, so a large value of $\mu$ is beneficial. On the other hand, choosing a good guess $x_{0}$ close to $x_{\mu}^{\star}$ provides a low value of $p_{p}\left(x_{\mu}^{\star}\right)=\frac{1}{2}\left\|x_{\mu}^{\star}-x_{0}\right\|_{\ell_{2}}^{2}$, also improving the rate of convergence. Warm-starting with $x_{0}$ from a previous solve not only changes the starting point of the algorithm, but it beneficially changes $p_{p}$ as well.

These two observations motivate the following continuation-like algorithm. 
Initialize $\mu_{0}, x_{0}$, and the number of continuation steps $T$. For $t=1, \ldots, T$,

1. Apply Nesterov's algorithm with $\mu=\mu^{(t)}$ and $x_{0}=x_{\mu^{(t-1)}}$.

2. Decrease the value of $\mu: \mu^{(t+1)}=\gamma \mu^{(t)}$ with $\gamma<1$.

Stop when the desired value of $\mu_{f}$ is reached.

This algorithm iteratively finds the solutions to a succession of problems with decreasing smoothing parameters $\mu_{0}>\cdots>\mu_{f}=\gamma^{T} \mu_{0}$ producing a sequence of-hopefully-finer estimates of $x_{\mu_{f}}^{\star} ;{ }^{6}$ these intermediate solutions are cheap to compute and provide a string of convenient first guesses for the next problem. In practice, they are solved with less accuracy, making them even cheaper to compute. This kind of continuation is different from standard continuation techniques [36, 49, 28, 58]. Indeed, while standard continuation solves a sequence of problem $\left(\mathrm{QP}_{\lambda}\right)$ with different values of the Lagrange multiplier $\lambda$ (so that intermediate solutions may have a meaningful interpretation if $\lambda$ is not decreased too quickly), the proposed continuation technique acts internally by changing the smoothing parameter $\mu$ while keeping $\epsilon$ and, therefore, the feasible set fixed. While intermediate solutions may have a less meaningful interpretation, the continuation may actually be more effective than traditional continuation since not only is the new step warm-started, but the primal prox-function is improved.

The value of $\mu_{f}$ is based on a desired accuracy as explained in section 3.5. As for an initial value $\mu_{0},(3.5)$ makes clear that the smoothing parameter plays a role similar to a threshold. A first choice may then be $\mu_{0}=0.9\left\|A^{*} b\right\|_{\ell \infty}$.

We illustrate the good behavior of the continuation-inspired algorithm by applying NESTA with continuation to solve a sparse reconstruction problem from partial frequency data. In this series of experiments, we assess the performance of NESTA while the dynamic range of the signals to be recovered increases.

The signals $x$ are $s$-sparse signals - that is, they have exactly $s$ nonzero components - of size $n=4,096$ and $s=m / 40$. Put $\Lambda$ for the indices of the nonzero entries of $x$; the amplitude of each nonzero entry is distributed uniformly on a logarithmic scale with a fixed dynamic range. Specifically, each nonzero entry is generated as follows:

$$
x[i]=\eta_{1}[i] 10^{\alpha \eta_{2}[i]},
$$

where $\eta_{1}[i]= \pm 1$ with probability $1 / 2$ (a random sign) and $\eta_{2}[i]$ is uniformly distributed in $[0,1]$. The parameter $\alpha$ quantifies the dynamic range. Unless specified otherwise, a dynamic range of $d \mathrm{~dB}$ means that $\alpha=d / 20$ (since for large signals $\alpha$ is approximately the logarithm base 10 of the ratio between the largest and lowest magnitudes). For instance, $80 \mathrm{~dB}$ signals are generated according to (3.15) with $\alpha=4$.

The measurements $A x$ consist of $m=n / 8$ random discrete cosine measurements so that $A^{*} A$ is diagonalized by the DCT. Finally, $b$ is obtained by adding a white Gaussian noise term with standard deviation $\sigma=0.1$. The initial value of the smoothing parameter is

\footnotetext{
${ }^{6}$ The parameter $\gamma$ does not define a new parameter; it depends upon $\mu_{f}, \mu_{0}$, and $T$ via $\gamma=\left(\mu_{f} / \mu_{0}\right)^{1 / T}$.
} 


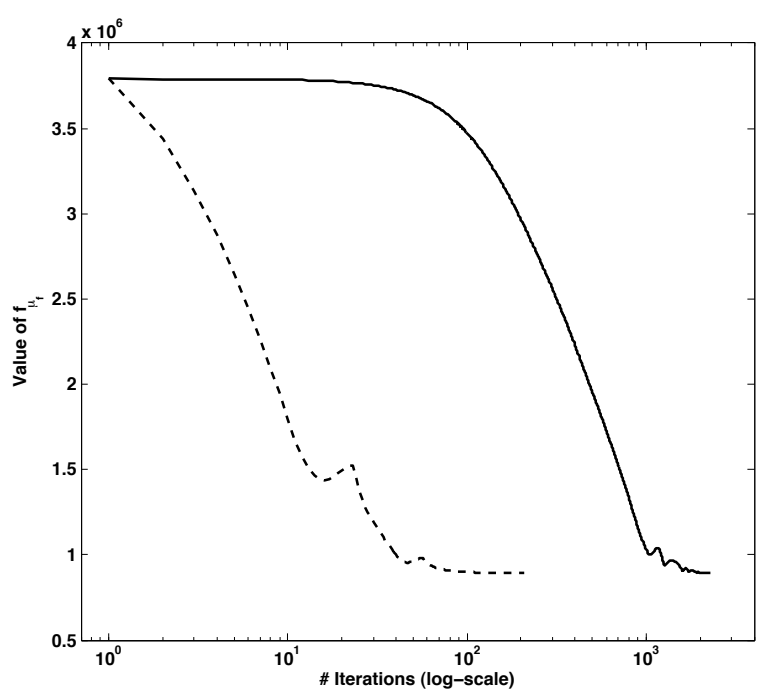

Figure 1. Value of $f_{\mu_{f}}\left(x_{k}\right)$ as a function of iteration $k$. Solid line: without continuation. Dashed line: with continuation. Here, the test signal has $100 \mathrm{~dB}$ of dynamic range.

$\mu_{0}=\left\|A^{*} b\right\|_{\ell_{\infty}}$, and the terminal value is $\mu_{f}=2 \sigma$. The algorithm terminates when the relative variation of $f_{\mu}$ is lower than $\delta=10^{-5}$. NESTA with continuation is applied to 10 random trials for a varying number of continuation steps $T$ and various values of the dynamic range. Figure 1 graphs the value of $f_{\mu_{f}}$ while applying NESTA with and without continuation as a function of the iteration count. The number of continuation steps is set to $T=4$.

One can observe that computing the solution to $\min f_{\mu_{f}}$ (solid line) takes a while when computed with the final value $\mu_{f}$; notice that NESTA seems to be slow at the beginning (number of iterations lower than 15). In the meantime NESTA with continuation rapidly estimates a sequence of coarse intermediate solutions that converges to the solution to $\min f_{\mu_{f}}$. In this case, continuation clearly enhances the global speed of convergence by a factor of 10 . Figure 2 provides deeper insights into the behavior of continuation with NESTA and shows the number of iterations required to reach convergence for varying values of the continuation steps $T$ for different values of the dynamic range.

When the ratio $\mu_{0} / \mu_{f}$ is low or when the required accuracy is low, continuation is not as beneficial: intermediate continuation steps require a number of iterations which may not speed up overall convergence. The step size which is about $L_{\mu_{f}}^{-1}$ works well in this regime. When the dynamic range increases and we require more accuracy, however, the ratio $\mu_{0} / \mu_{f}$ is large, since $\mu_{0}=.9\left\|A^{*} b\right\|_{\ell_{\infty}} \approx\|x\|_{\ell_{\infty}} \gg \sigma$, and continuation provides considerable improvements. In this case, the step size $L_{\mu_{f}}^{-1}$ is too conservative, and it takes a while to find the large entries of $x$. Empirically, when the dynamic range is $100 \mathrm{~dB}$, continuation improves the speed of convergence by a factor of 8 . As this factor is likely to increase exponentially with the dynamic range (when expressed in dB), NESTA with continuation seems to be a better candidate for solving sparse reconstruction problems with high accuracy.

Interestingly, the behavior of NESTA with continuation seems to be quite stable: increasing the number of continuation steps does not increase dramatically the number of iterations. In practice, although the ideal $T$ is certainly signal dependent, we have observed in our prob- 


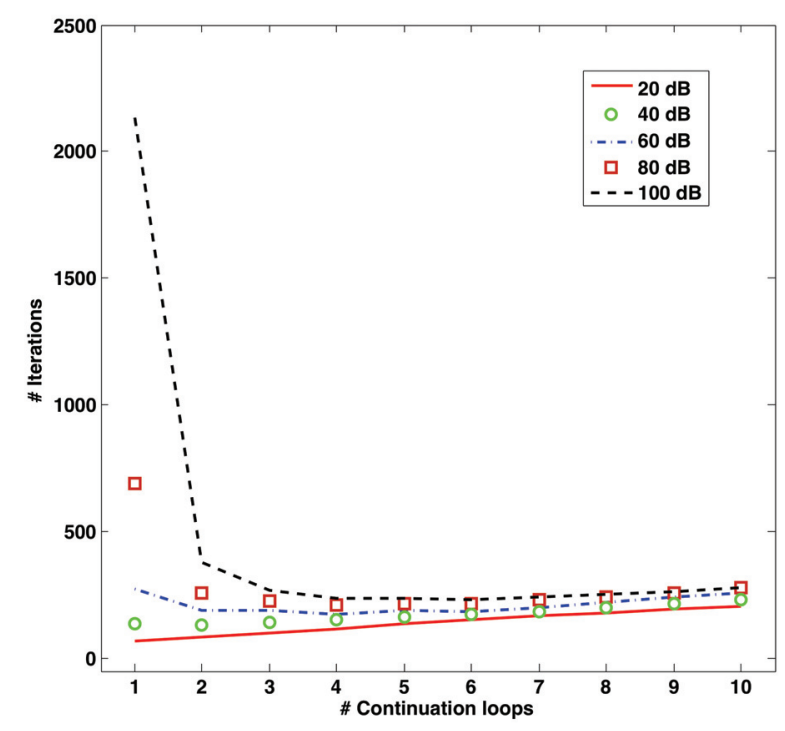

Figure 2. Total number of iterations required for convergence with a varying number of continuation steps and for different values of the dynamic range.

lem settings that choosing $T \in\{4,5,6\}$ leads to reasonable results. The value of $T$ should certainly be optimized for particular problem types.

3.7. Some theoretical considerations. The convergence of NESTA with and without continuation is straightforward. The following theorem states that at each continuation step with $\mu=\mu^{(t)}$, the sequence $f_{\mu}\left(y_{k}\right)$ converges to $f_{\mu}\left(x_{\mu}^{\star}\right)$. Global convergence is proved by applying this theorem to $t=T$.

Theorem 3.1. At each continuation step $t, \lim _{k \rightarrow \infty} f_{\mu^{(t)}}\left(y_{k}\right)=f_{\mu^{(t)}}\left(x_{\mu^{(t)}}^{\star}\right)$, and

$$
f_{\mu^{(t)}}\left(y_{k}\right)-f_{\mu^{(t)}}\left(x_{\mu^{(t)}}^{\star}\right) \leq \frac{2 L_{\mu^{(t)}}\left\|x_{\mu^{(t)}}^{\star}-x_{\mu^{(t-1)}}\right\|_{\ell_{2}}^{2}}{k^{2}} .
$$

Proof. The proof is immediate by using [47, Theorem 2].

As mentioned earlier, continuation may be valuable for improving the speed of convergence. Let each continuation step $t$ stop after $\mathcal{N}^{(t)}$ iterations with

$$
\mathcal{N}^{(t)}=\sqrt{\frac{2 L_{\mu^{(t)}}}{\gamma^{t} \delta_{0}}}\left\|x_{\mu^{(t)}}^{\star}-x_{\mu^{(t-1)}}^{\star}\right\|_{\ell_{2}}
$$

so that we have

$$
f_{\mu^{(t)}}\left(y_{k}\right)-f_{\mu^{(t)}}\left(x_{\mu^{(t)}}^{\star}\right) \leq \gamma^{t} \delta_{0},
$$

where the accuracy $\gamma^{t} \delta_{0}$ becomes tighter as $t$ increases. Then summing up the contribution of all the continuation steps gives

$$
\mathcal{N}_{c}=\sqrt{\frac{2}{\mu_{0} \delta_{0}}} \sum_{t=1}^{T} \gamma^{-t}\left\|x_{\mu^{(t)}}^{\star}-x_{\mu^{(t-1)}}^{\star}\right\|_{\ell_{2}} .
$$

Copyright $(\odot$ by SIAM. Unauthorized reproduction of this article is prohibited. 

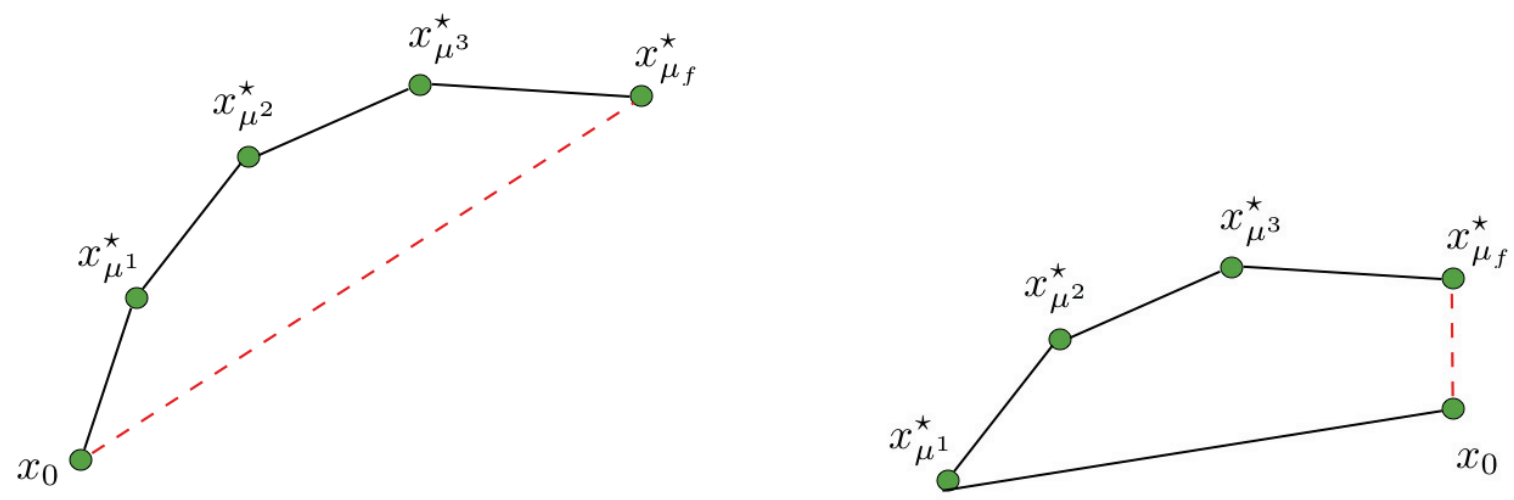

Figure 3. Typical solution paths. Left: smooth solution path. Right: nonsmooth solution path.

When NESTA is applied without continuation, the number of iterations required to reach convergence is

$$
\mathcal{N}=\sqrt{\frac{2}{\mu_{0} \delta_{0}}} \gamma^{-T}\left\|x_{\mu_{f}}^{\star}-x_{0}\right\|_{\ell_{2}}
$$

Now the ratio $\mathcal{N}_{c} / \mathcal{N}$ is given by

$$
\frac{\mathcal{N}_{c}}{\mathcal{N}}=\sum_{t=1}^{T} \gamma^{T-t} \frac{\left\|x_{\mu^{(t)}}^{\star}-x_{\mu^{(t-1)}}^{\star}\right\|_{\ell_{2}}}{\left\|x_{\mu_{f}}^{\star}-x_{0}\right\|_{\ell_{2}}} .
$$

Continuation is definitely worthwhile when the right-hand side is smaller than 1 . Interestingly, this quantity is directly linked to the path followed by the sequence $x_{0} \rightarrow x_{\mu^{(1)}} \rightarrow \cdots \rightarrow x_{\mu_{f}}$. More precisely, it is related to the smoothness of this path; for instance, if all the intermediate points $x_{\mu^{(t)}}$ belong to the segment $\left[x_{0}, x_{\mu_{f}}\right]$ in an ordered fashion, then $\sum_{t}\left\|x_{\mu^{(t)}}-x_{\mu^{(t-1)}}\right\|_{\ell_{2}}=$ $\left\|x_{\mu_{f}}^{\star}-x_{0}\right\|_{\ell_{2}}$. Hence, $\frac{\mathcal{N}_{c}}{\mathcal{N}}<1$, and continuation improves the convergence rate.

Figure 3 illustrates two typical solution paths with continuation. When the sequence of solutions obeys $\left\|x_{0}\right\|_{\ell_{1}} \geq \cdots \geq\left\|x_{\mu^{(t)}}^{\star}\right\|_{\ell_{1}} \geq \cdots \geq\left\|x_{\mu_{f}}^{\star}\right\|_{\ell_{1}}$ (this is the case when $x_{0}=A^{*} b$ and $\mu_{1} \geq \cdots \geq \mu^{(t)} \geq \cdots \geq \mu_{f}$ ), the solution path is likely to be "smooth"; that is, the solutions obey $\left\|x_{\mu^{(t)}}^{\star}-x_{\mu_{f}}^{\star}\right\|_{\ell_{2}} \geq\left\|x_{\mu^{(t+1)}}^{\star}-x_{\mu_{f}}^{\star}\right\|_{\ell_{2}}$ as on the left-hand side of Figure 3. The "nonsmooth" case on the right-hand side of Figure 3 arises when the sequence of smoothing parameters does not provide estimates of $x_{\mu_{f}}^{\star}$ that are all better than $x_{0}$. Here, computing some of the intermediate points $\left\{x_{\mu^{(t)}}^{\star}\right\}$ is wasteful, and continuation fails to be faster.

4. Accurate optimization. A significant portion of the numerical part of this paper focuses on comparing different sparse recovery algorithms in terms of speed and accuracy. In this section, we first demonstrate that NESTA can easily recover the exact solution to $\left(\mathrm{BP}_{\epsilon}\right)$ with a precision of 5-6 digits. Speaking of precision, we shall essentially use 2 criteria to evaluate accuracy. 
Table 1

Assessing the accuracy of the fast iterative soft-thresholding algorithm (FISTA) and NESTA when the optimal solution is known. The relative error on the optimal value is given by (4.1) and the $\ell_{\infty}$ error on the optimal solution by (4.2). $\mathcal{N}_{A}$ is the number of calls to $A$ or $A^{*}$ to compute the solution.

\begin{tabular}{l||c|c|c|c}
\hline Method & $\ell_{1}$ norm & Rel. error $\ell_{1}$ norm & $\ell_{\infty}$ error & $\mathcal{N}_{A}$ \\
\hline$x^{\star}$ & $3.33601 \mathrm{e}+6$ & & & \\
\hline FISTA & $3.33610 \mathrm{e}+6$ & $2.7 \mathrm{e}-5$ & 0.31 & 40000 \\
NESTA $\mu=0.02$ & $3.33647 \mathrm{e}+6$ & $1.4 \mathrm{e}-4$ & 0.08 & 513 \\
\hline
\end{tabular}

1. The first is the (relative) error on the objective functional

$$
\frac{\|x\|_{\ell_{1}}-\left\|x^{\star}\right\|_{\ell_{1}}}{\left\|x^{\star}\right\|_{\ell_{1}}}
$$

where $x^{\star}$ is the optimal solution to $\left(\mathrm{BP}_{\epsilon}\right)$.

2. The second is the accuracy of the optimal solution itself and is measured via

$$
\left\|x-x^{\star}\right\|_{\ell_{\infty}},
$$

which gives a precise value of the accuracy per entry.

4.1. Is NESTA accurate? For general problem instances, the exact solution to $\left(\mathrm{BP}_{\epsilon}\right)$ (or, equivalently, $\left(\mathrm{QP}_{\lambda}\right)$ ) cannot be computed analytically. Under some conditions, however, a simple formula is available when the optimal solution has exactly the same support and the same sign as the unknown (sparse) $x^{0}$ (recall the model $b=A x^{0}+z$ ). Denote by $I$ the support of $x^{0}, I:=\left\{i:\left|x^{0}[i]\right|>0\right\}$. Then if $x^{0}$ is sufficiently sparse and if the nonzero entries of $x^{0}$ are sufficiently large, the solution $x^{\star}$ to $\left(\mathrm{QP}_{\lambda}\right)$ is given by

$$
\begin{aligned}
x^{\star}[I] & =\left(A[I]^{*} A[I]\right)^{-1}\left(A[I]^{*} b-\lambda \operatorname{sgn}\left(x^{0}[I]\right)\right), \\
x^{\star}\left[I^{c}\right] & =0 ;
\end{aligned}
$$

see [12], for example. In this expression, $x[I]$ is the vector with indices in $I$, and $A[I]$ is the submatrix with columns indices in $I$.

To evaluate NESTA's accuracy, we set $n=262,144, m=n / 8$, and $s=m / 100$ (this is the number of nonzero coordinates of $x_{0}$ ). The absolute values of the nonzero entries of $x_{0}$ are distributed between 1 and $10^{5}$ so that we have about $100 \mathrm{~dB}$ of dynamic range. The measurements $A x^{0}$ are discrete cosine coefficients selected uniformly at random. We add Gaussian white noise with standard deviation $\sigma=0.01$. We then compute the solution (4.3) and make sure it obeys the KKT optimality conditions for $\left(\mathrm{QP}_{\lambda}\right)$ so that the optimal solution is known.

We run NESTA with continuation with the value of $\epsilon:=\left\|b-A x^{\star}\right\|$. We use $\mu=0.02$ and $\delta=10^{-7}$, and the number of continuation steps is set to 5 . Table 1 reports on numerical results. First, the value of the objective functional is accurate up to 4 digits. Second, the computed solution is very accurate since we observe an $\ell_{\infty}$ error of 0.08 . Now recall that the nonzero components of $x^{\star}$ vary from about 1 to $10^{5}$ so that we have high accuracy over a huge dynamic range. This can also be gleaned from Figure 4, which plots NESTA's solution versus the optimal solution, and confirms the excellent precision of our algorithm.

Copyright $\odot$ by SIAM. Unauthorized reproduction of this article is prohibited. 


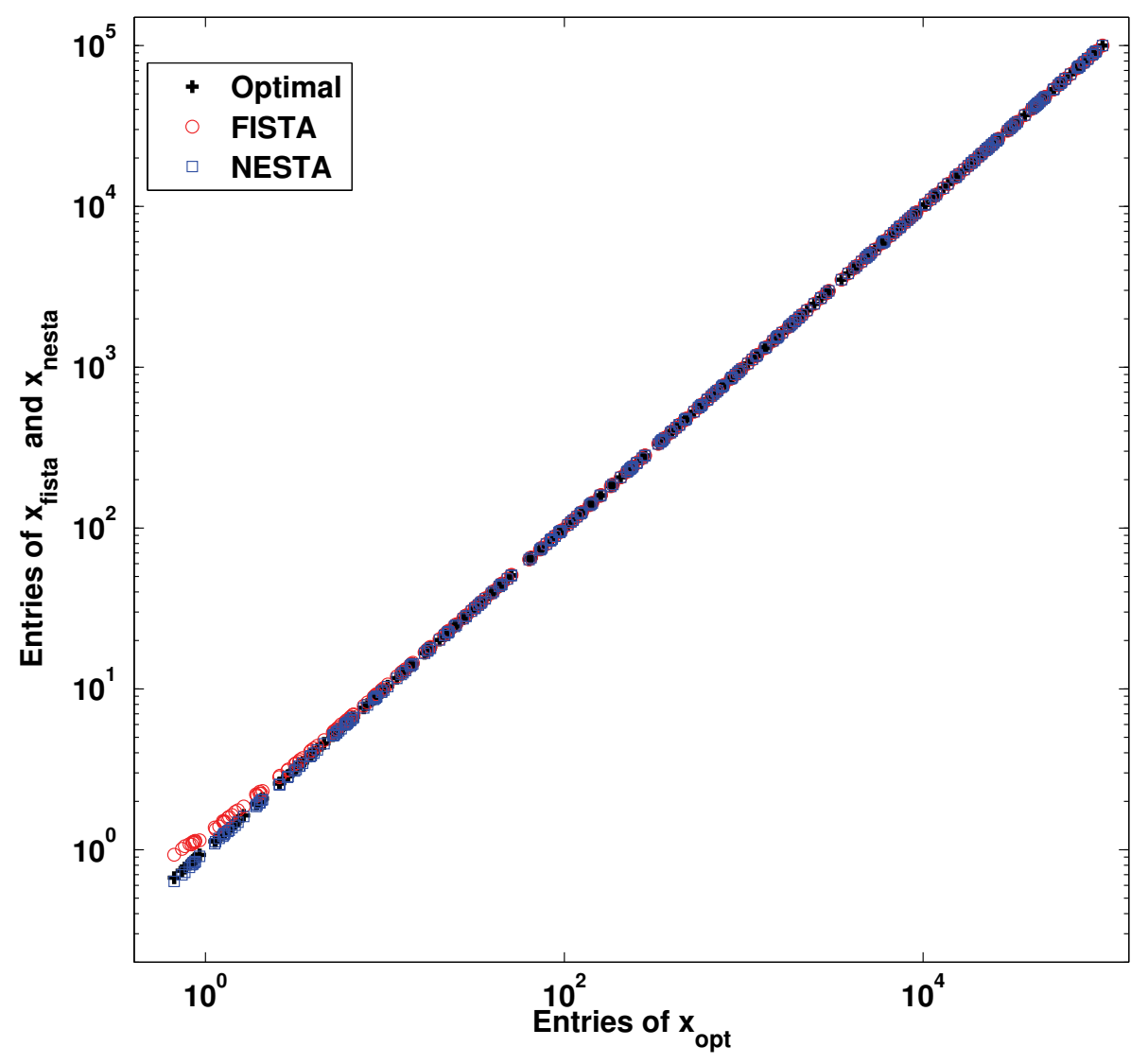

Figure 4. Entries of the computed solutions versus the optimal solution. The absolute values of the entries on the support of the optimal solution are plotted.

4.2. Setting up a reference algorithm for accuracy tests. In general situations, a formula for the optimal solution is, of course, unavailable, and evaluating the accuracy of solutions requires defining a method of reference. In this paper, we will use FISTA [4] as such a reference since it is an efficient algorithm that also turns out to be extremely easy to use; in particular, no parameter has to be tweaked, except for the standard stopping criterion (maximum number of iterations and tolerance on the relative variation of the objective function).

We run FISTA with 20,000 iterations on the same problem as above and report its accuracy in Table 1. The $\ell_{1}$ norm is exact up to 4 digits. Furthermore, Figure 4 shows the entries of FISTA's solution versus those of the optimal solution, and one observes a very good fit (near perfect when the magnitude of a component of $x^{\star}$ is higher than 3 ). The $\ell_{\infty}$ error between FISTA's solution and the optimal solution $x^{\star}$ is equal to 0.31 ; that is, the entries are exact up to \pm 0.31 . Because this occurs over an enormous dynamic range, we conclude that FISTA also gives very accurate solutions, provided that sufficiently many iterations are taken. We have observed that running FISTA with a high number of iterations - typically greater than 20,000 - provides accurate solutions to $\left(\mathrm{QP}_{\lambda}\right)$, and this is why we will use it as our method of reference in the forthcoming comparisons in this section and the next. 
4.3. The smoothing parameter $\mu$ and NESTA's accuracy. By definition, $\mu$ fixes the accuracy of the approximation $f_{\mu}$ to the $\ell_{1}$ norm, and, therefore, NESTA's accuracy directly depends on this parameter. We now propose assessing the accuracy of NESTA for different values of $\mu$. The problem sizes are as before, namely, $n=262,144$ and $m=n / 8$, except that now the unknown $x^{0}$ is far less sparse with $s=m / 5$. The standard deviation of the additive Gaussian white noise is also higher, and we set $\sigma=0.1$.

Because of the larger values of $s$ and $\sigma$, it is no longer possible to have an analytic solution from (4.3). Instead, we use FISTA to compute a reference solution $x_{F}$, using 20,000 iterations and with $\lambda=0.0685$, which gives $\left\|b-A x_{F}\right\|_{\ell_{2}}^{2} \simeq(m+2 \sqrt{2 m}) \sigma^{2}$. To be sure that FISTA's solution is very close to the optimal solution, we check that the KKT stationarity condition is nearly verified. If $I_{\star}$ is the support of the optimal solution $x^{\star}$, this condition reads as

$$
\begin{aligned}
A\left[I_{\star}\right]^{*}\left(b-A x^{\star}\right) & =\lambda \operatorname{sgn}\left(x^{\star}\left[I_{\star}\right]\right), \\
\left\|A\left[I_{\star}^{c}\right]^{*}\left(b-A x^{\star}\right)\right\|_{\ell_{\infty}} & \leq \lambda .
\end{aligned}
$$

Now define $I$ to be the support of $x_{F}$. Then, here, $x_{F}$ obeys

$$
\begin{aligned}
\left\|A[I]^{*}\left(b-A x_{F}\right)-\lambda \operatorname{sgn}\left(x_{F}[I]\right)\right\|_{\ell_{\infty}} & =\left(2.66 \times 10^{-10}\right) \lambda, \\
\left\|A\left[I^{c}\right]^{*}\left(b-A x_{F}\right)\right\|_{\ell_{\infty}} & \leq 0.99 \lambda .
\end{aligned}
$$

This shows that $x_{F}$ is extremely close to the optimal solution.

NESTA is run with $T=5$ continuation steps for three different values of $\mu \in\{0.2,0.02,0.002\}$ (the tolerance $\delta$ is set to $10^{-6}, 10^{-7}$, and $10^{-8}$, respectively). Figure 5 plots the solutions given by NESTA versus the "optimal solution" $x_{F}$. Clearly, when $\mu$ decreases, the accuracy of NESTA increases just as expected. More precisely, notice in Table 2 that for this particular experiment, decreasing $\mu$ by a factor of 10 gives about 1 additional digit of accuracy on the optimal value.

According to this table, $\mu=0.02$ seems a reasonable choice for this particular signal to guarantee an accurate solution since one has between 4 and 5 digits of accuracy on the optimal value, and since the $\ell_{\infty}$ error is lower than 1 . Observe in Figure 5 that this value separates the nonzero entries from the noise floor (when $\sigma=0.01$ ). In the extensive numerical experiments of section 5 , we shall set $\mu=0.02$ and $\delta=10^{-7}$ as default values.

5. Numerical comparisons. This section presents numerical experiments comparing several state-of-the-art optimization techniques designed to solve (1.2) or (1.3). To be as fair as possible, we propose comparisons with methods for which software is publicly available online.

Some extensive numerical results have been reported in [55, 58, 40,41], but to the best of our knowledge, extensive tests comparing algorithms for solving $\left(\mathrm{QP}_{\lambda}\right)$ and $\left(\mathrm{BP}_{\epsilon}\right)$ are currently unavailable, and one novelty of our experimental study is that it uses fair stopping criteria introduced in section 5.3. Moreover, whereas publications sometimes test algorithms on relatively easy and academic problems, we will subject optimization methods to hard but realistic $\ell_{1}$ reconstruction problems.

In our view, a challenging problem involves some or all of the characteristics below.

1. High dynamic range. As mentioned earlier, most optimization techniques are able to find (more or less rapidly) the most significant entries (those with a large amplitude) of the

Copyright (c) by SIAM. Unauthorized reproduction of this article is prohibited. 


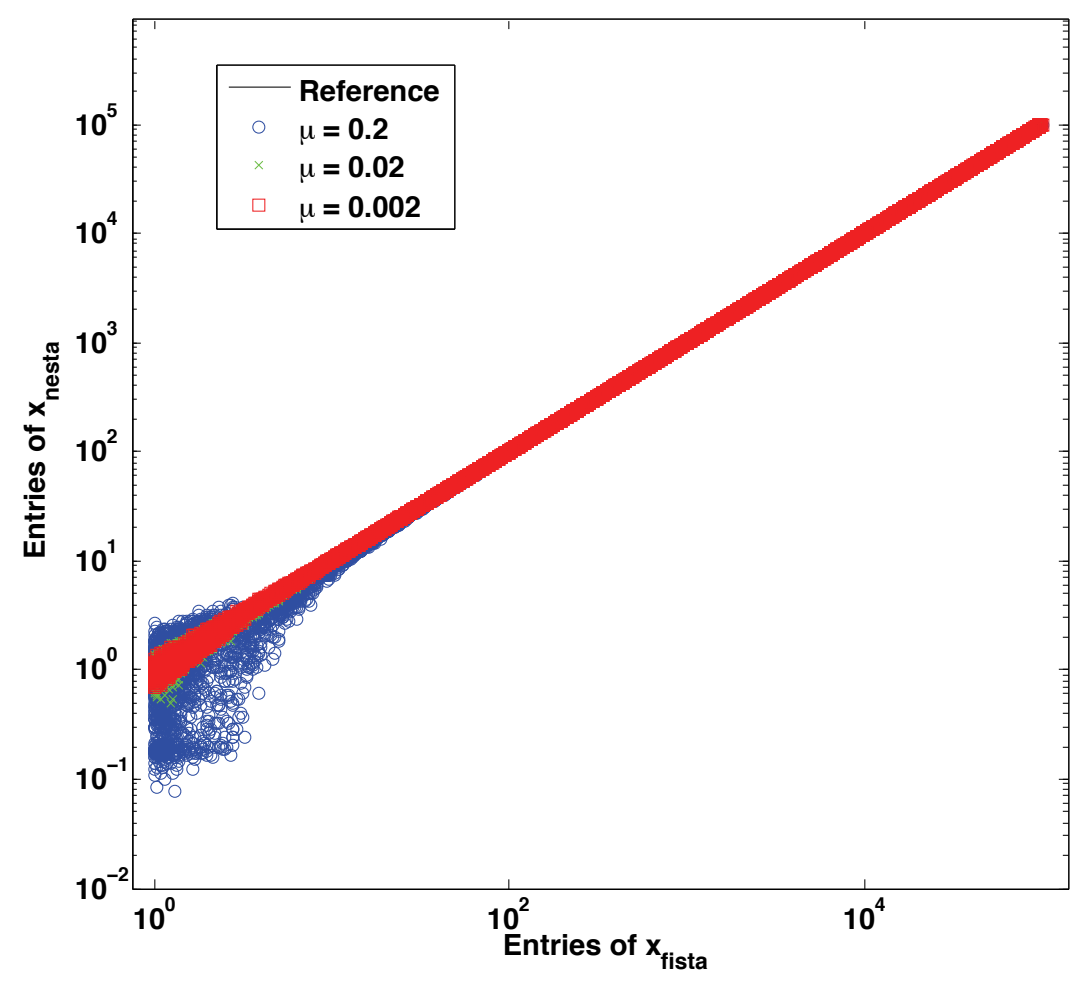

Figure 5. Entries of the computed solutions versus the optimal solution. We plot the absolute values of the entries on the set where the magnitude of the optimal solution exceeds 1.

Table 2

NESTA's accuracy. The errors and number of function calls $\mathcal{N}_{A}$ have the same meaning as in Table 1.

\begin{tabular}{l||c|c|c|c}
\hline Method & $\ell_{1}$ norm & Rel. error $\ell_{1}$ norm & $\ell_{\infty}$ error & $\mathcal{N}_{A}$ \\
\hline FISTA & $5.71539 \mathrm{e}+7$ & & & \\
\hline NESTA $\mu=0.2$ & $5.71614 \mathrm{e}+7$ & $1.3 \mathrm{e}-4$ & 3.8 & 659 \\
NESTA $\mu=0.02$ & $5.71547 \mathrm{e}+7$ & $1.4 \mathrm{e}-5$ & 0.96 & 1055 \\
NESTA $\mu=0.002$ & $5.71540 \mathrm{e}+7$ & $1.6 \mathrm{e}-6$ & 0.64 & 1537 \\
\hline
\end{tabular}

signal $x$. Accurately recovering the entries of $x$ that have low magnitudes is more challenging. Methods that can accurately recover high dynamic range signals are of crucial importance in some real-world applications; see section 6.1.

2. Approximate sparsity. Realistic signals are seldom exactly sparse, and, therefore, coping with approximately sparse signals is of paramount importance. In signal or image processing, for example, wavelet coefficients of natural images contain many low level entries that are worth retrieving.

3. Large scale. Some standard optimization techniques, such as interior point methods, are known to provide accurate solutions. However, these techniques are not applicable to largescale problems due to the large cost of solving linear systems. Furthermore, many existing software packages fail to take advantage of fast algorithms for applying $A$. We will focus on 
large-scale problems in which the number of unknowns $n$ is more than a quarter of a million, i.e., $n=262,144$.

5.1. State-of-the-art methods. Most of the algorithms discussed in this section are considered to be state-of-art in the sense that they are the most competitive among sparse reconstruction algorithms. Some of these methods have been improved after some years of research $[38,33]$. Others make careful use of the fact that $\ell_{1}$ minimization produces sparse solutions, and activate special code when they estimate that a fixed active set has been found $[55,57]$. Finally, our focus is on rapid algorithms, so we are interested in methods which can take advantage of fast algorithms for applying $A$ to a vector. This is why we have not tested other good methods such as that in [34], for example.

Most of the following algorithms solve $\left(\mathrm{QP}_{\lambda}\right)$ and require gradient or subgradient information at the current iterate. Because of the quadratic penalty, the gradient requires one application of $A$ and one of $A^{*}$, so the dominant cost per iteration is $2 \mathcal{C}_{A}$.

5.1.1. NESTA. Below, we apply NESTA with the default parameters

$$
x_{0}=A^{*} b, \quad \mu=0.02, \quad \delta=10^{-7}
$$

(recall that $x_{0}$ is the initial guess). The maximal number of iterations is set to $\mathcal{I}_{\max }=10,000$; if convergence is not reached after $\mathcal{I}_{\max }$ iterations, we record that the algorithm did not converge (DNC). Because NESTA requires 2 calls to either $A$ or $A^{*}$ per iteration, this is equivalent to declaring DNC after $\mathcal{N}_{A}=20,000$ iterations, where $\mathcal{N}_{A}$ refers to the total number of calls to $A$ or $A^{*}$; hence, for the other methods, we declare DNC when $\mathcal{N}_{A}>20,000$. When continuation is used, extra parameters are set up as follows:

$$
T=4, \quad \mu_{0}=\left\|x_{0}\right\|_{\ell_{\infty}}, \quad \gamma=\left(\mu / \mu_{0}\right)^{1 / T},
$$

and for $t=1, \ldots, T$,

$$
\mu_{t}=\gamma^{t} \mu_{0}, \quad \delta_{t}=0.1 \cdot(\delta / 0.1)^{t / T},
$$

where $\delta_{t}$ fixes the stopping criterion in the $t$ th continuation step. Numerical results are reported and discussed in section 5.4.

5.1.2. Gradient projections for sparse reconstruction (GPSR) [33]. GPSR has been introduced in [33] to solve the standard $\ell_{1}$ minimization problem in Lagrangian form $\left(\mathrm{QP}_{\lambda}\right)$. GPSR uses a standard linear programming change-of-variables trick to recast the variable $x=v_{1}-v_{2}$, with the requirement that $v_{1}, v_{2} \geq 0$. This is a different problem, but it clearly has the same solution set. The advantage is that the $\ell_{1}$ norm is replaced by a linear functional, so that the objective is now smooth. Projecting $v_{1}$ and $v_{2}$ onto the nonnegative orthant is trivial. Different techniques for choosing the step size $\alpha_{k}$ (backtracking, Barzilai-Borwein [3], and so on) are discussed in [33]. The code is available at http://www.lx.it.pt/ mtf/GPSR/. In the forthcoming experiments, the parameters are set to their default values.

GPSR also implements continuation, and we tested this version as well. All parameters were set to defaults except that, per the recommendation of one of the GPSR authors to increase performance, the number of continuation steps was set to 40 , the ToleranceA variable was set to $10^{-3}$, and the MiniterA variable was set to 1 . In addition, the code itself was

Copyright (c) by SIAM. Unauthorized reproduction of this article is prohibited. 
tweaked a bit; in particular, the stopping criterion for continuation steps (other than the final step) was changed. Future releases of GPSR will probably contain a similarly updated continuation stopping criterion.

5.1.3. Sparse reconstruction by separable approximation (SpaRSA) [58]. SpaRSA is an algorithm for minimizing composite functions $\phi(x)=f(x)+\lambda c(x)$ composed of a smooth term $f$ and a separable nonsmooth term $c$, e.g., $\left(\mathrm{QP}_{\lambda}\right)$. At every step, a subproblem of the form

$$
\text { minimize }\|x-y\|_{\ell_{2}}^{2}+\frac{\lambda}{\alpha} c(x)
$$

with optimization variable $x$ must be solved; this is the same as computing the proximity operator corresponding to $c$. For $\left(\mathrm{QP}_{\lambda}\right)$, the solution is given by shrinkage. In this sense, SpaRSA is an iterative shrinkage/thresholding (IST) algorithm, much like FISTA (though without the accelerated convergence) and fixed point continuation (FPC). Also like FPC, continuation is used to speed convergence, and like FPC-BB, a Barzilai-Borwein heuristic is used for the step size. The code for SpaRSA may be obtained at http://www.lx.it.pt/ mtf/SpaRSA/. Parameters were set to default except that the number of continuation steps was set to 40 and the MiniterA variable was set to 1 (instead of the default 5), as per the recommendations of one of the SpaRSA authors - again, to increase performance.

5.1.4. $\ell_{1}$ regularized least squares (I1_Is) [38]. This method solves the standard unconstrained $\ell_{1}$ minimization problem and is an interior point method (with log-barrier) using preconditioned conjugate gradient (PCG) to accelerate convergence and stabilize the algorithm. The preconditioner used in the PCG step is a linear combination of the diagonal approximation of the Hessian of the quadratic term and of the Hessian of the log-barrier term. 11_ls is shown to be faster than usual interior point methods; nevertheless, each step requires solving a linear system of the form $H \Delta x=g$. Even if PCG makes the method more reliable, 11_ls is still problematic for large-scale problems. In the next comparisons, we provide some typical values of its computational complexity compared to those of the other methods. The code is available at http://www.stanford.edu/ boyd/11_ls/.

5.1.5. Spectral projected gradient (SPGL1) [55]. In 2008, Van Den Berg and Friedlander [55] adapted the spectral projected gradient algorithm introduced in [6] to solve the LASSO $\left(\mathrm{LS}_{\tau}\right)$. Interestingly, they introduced a clever root finding procedure such that solving a few instances of $\left(\mathrm{LS}_{\tau}\right)$ for different values of $\tau$ enabled them to equivalently solve $\left(\mathrm{BP}_{\epsilon}\right)$. Furthermore, if the algorithm detects a nearly sparse solution, it defines an active set and solves an equation like (4.3) on this active set. In the next experiments, the parameters are set to their default values. The code is available at http://www.cs.ubc.ca/labs/scl/spgl1/.

5.1.6. Fixed point continuation method $[36,37]$. The FPC method is a recent firstorder algorithm for solving $\left(\mathrm{QP}_{\lambda}\right)$ and simple generalizations of $\left(\mathrm{QP}_{\lambda}\right)$. The main idea is based on a fixed point equation, $x=F(x)$, which holds at the solution (derived from the subgradient optimality condition, where $F$ is a composition of shrinkage and a gradient step). For appropriate parameters, $F$ is a contraction, and thus the algorithm $x_{k+1}=F\left(x_{k}\right)$ converges ( $q$-linearly). The parameter $\lambda$ in $\left(\mathrm{QP}_{\lambda}\right)$ determines the amount of shrinkage and, therefore, the speed of convergence; thus in practice, $\lambda$ is decreased in a continuation scheme. Code

Copyright (c) by SIAM. Unauthorized reproduction of this article is prohibited. 
for FPC is available at http://www.caam.rice.edu/ optimization/L1/fpc/. Also available is a state-of-the-art version of FPC from 2008 that uses Barzilai-Borwein [3] steps to accelerate performance. In the numerical tests, the Barzilai-Borwein version (referred to as FPC-BB) significantly outperforms standard FPC. All parameters were set to default values.

5.1.7. FPC active set (FPC-AS) [57]. In 2009, inspired by both first-order algorithms, such as FPC, and greedy algorithms [29, 44], Wen et al. [57] extended FPC into the two-part algorithm FPC-AS to solve $\left(\mathrm{QP}_{\lambda}\right)$. In the first stage, FPC-AS calls an improved version of FPC that allows the step size to be updated dynamically, using a nonmonotone exact line search to ensure $r$-linear convergence and also incorporating a Barzilai-Borwein [3] heuristic. After a given stopping criterion, the current value, $x_{k}$, is hard-thresholded to determine an active set. On the active set, $\|x\|_{\ell_{1}}$ is replaced by $c^{*} x$, where $c=\operatorname{sgn}\left(x_{k}\right)$, with the constraints that $x[i] \cdot c[i]>0$ for all the indices $i$ belonging to the active set. This subproblem has a smooth objective, so it can be solved using smooth optimization techniques. This two-step process is then repeated for a smaller value of $\lambda$ in a continuation scheme. We tested FPC-AS using both L-BFGS (the default) and CG (which we refer to as FPC-AS-CG) to solve the subproblem; neither of these solvers actually enforces the $x[i] \cdot c[i]>0$ constraint on the active set. Code for FPC-AS is available at http://www.caam.rice.edu/ optimization/L1/FPC_AS/.

For $s$-sparse signals, all parameters were set to defaults except for the stopping criteria (as discussed in section 5.3). For approximately sparse signals, FPC-AS performed poorly ( $>10,000$ iterations) with the default parameters. By changing a parameter that controls the estimated number of nonzeros from $m / 2$ (default) to $n$, the performance improved dramatically, and this is the performance reported in Tables 5-6. The maximum number of subspace iterations was also changed from the default to 10, as recommended in the help file.

5.1.8. Bregman. The Bregman iterative algorithm, motivated by the Bregman distance, has been shown to be surprisingly simple [60]. The first iteration solves $\left(\mathrm{QP}_{\lambda}\right)$ for a specified value of $\lambda$; subsequent iterations solve $\left(\mathrm{QP}_{\lambda}\right)$ for the same value of $\lambda$, with an updated observation vector $b$. Typically, only a few outer iterations are needed (e.g., 4), but each iteration requires a solve of $\left(\mathrm{QP}_{\lambda}\right)$, which is costly. The original Bregman algorithm calls FPC to solve these subproblems; we test Bregman using FPC and FPC-BB as subproblem solvers.

A version of the Bregman algorithm, known as the linearized Bregman algorithm [50, 9], takes only one step of the inner iteration per outer iteration; consequently, many outer iterations are taken, in contrast to the regular Bregman algorithm. It can be shown that linearized Bregman is equivalent to gradient ascent on the dual problem. Linearized Bregman was not included in the tests because no standardized public code is available. Code for the regular Bregman algorithm may be obtained at http://www.caam.rice.edu/ optimization/L1/ bregman/. There are quite a few parameters, since there are parameters for the outer iterations and for the inner (FPC) iterations; for all experiments, parameters were set to defaults. In particular, we noted that using the default stopping criteria for the inner solve, which limited FPC to 1,000 iterations, led to significantly better results than allowing the subproblem to run to 10,000 iterations.

5.1.9. Fast iterative soft-thresholding algorithm (FISTA) [4]. FISTA is based upon Nesterov's work, but departs from NESTA in two important ways: (1) FISTA solves the sparse unconstrained reconstruction problem $\left(\mathrm{QP}_{\lambda}\right) ;(2)$ FISTA is a proximal subgradient algorithm,

Copyright (c) by SIAM. Unauthorized reproduction of this article is prohibited. 
which uses only two sequences of iterates. In some sense, FISTA is a simplified version of the algorithm previously introduced by Nesterov to minimize composite functions [48]. The theoretical rate of convergence of FISTA is $\mathcal{O}\left(1 / k^{2}\right)$.

For each test, FISTA is run twice: it is first run until the relative variation in the function value is less than $10^{-14}$, with no limit on function calls, and this solution is used as the reference solution. The second time, it is run using the same stopping criteria as the other algorithms, as explained in section 5.3.

5.2. Constrained versus unconstrained minimization. We would like to briefly highlight the fact that these algorithms are not solving the same problem. SPGL1 solves the constrained problem $\left(\mathrm{BP}_{\epsilon}\right)$, and NESTA solves an approximate solution to $\left(\mathrm{BP}_{\epsilon}\right)$, while all other methods tested solve the unconstrained problem $\left(\mathrm{QP}_{\lambda}\right)$ - NESTA and SPGL1 can also solve $\left(\mathrm{QP}_{\lambda}\right)$. Solving a constrained problem may sometimes be more challenging than similar unconstrained problems (witness the popularity of penalty functions and augmented Lagrangian methods), and given the numerous algorithms for solving $\left(\mathrm{QP}_{\lambda}\right)$ and the relatively few algorithms for solving $\left(\mathrm{BP}_{\epsilon}\right)$, it empirically seems that $\left(\mathrm{BP}_{\epsilon}\right)$ is the harder problem. ${ }^{7}$ For example, it may be hard to even find a feasible point for $\left(\mathrm{BP}_{\epsilon}\right)$, since the pseudoinverse of $A$, when $A$ is not a projection, may be difficult to compute. Thus, we emphasize that SPGL1 and NESTA are actually more general than the other algorithms (and as section 6 shows, NESTA is even more general because it handles a wide variety of constrained problems); this is especially important because from a practical viewpoint, it may be easier to estimate an appropriate $\epsilon$ than an appropriate value of $\lambda$. Furthermore, as will be shown in section 5.4, SPGL1 and NESTA with continuation are also the most robust methods for arbitrary signals (i.e., they perform well even when the signal is not exactly sparse and even when it has high dynamic range). Combining these two facts, we feel that these two algorithms are extremely useful for real-world applications.

5.3. Experimental protocol. In these experiments, we compare NESTA with other efficient methods. There are two main difficulties with comparisons, which might explain why broad comparisons have not been offered before. The first problem is that some algorithms, such as NESTA, solve $\left(\mathrm{BP}_{\epsilon}\right)$, whereas other algorithms solve $\left(\mathrm{QP}_{\lambda}\right)$. Given $\epsilon$, it is difficult to compute a $\lambda(\epsilon)$ that gives an equivalence between the problems; in theory, the KKT conditions give $\lambda$, but we have observed in practice that because we have an approximate solution (albeit a very accurate one), computing $\lambda$ in this fashion is not stable.

Instead, we note that, given $\lambda$ and a solution $x_{\lambda}$ to $\left(\mathrm{QP}_{\lambda}\right)$, it is easy to compute a very accurate $\epsilon(\lambda)$ since $\epsilon=\left\|A x_{\lambda}-b\right\|_{\ell_{2}}$. Hence, we use a two-step procedure. In the first step, we choose a value of $\epsilon_{0}=\sqrt{m+2 \sqrt{2 m}} \sigma$ based on the noise level $\sigma$ (since a value of $\lambda$ that corresponds to $\sigma$ is less clear), and use SPGL1 to solve $\left(\mathrm{BP}_{\epsilon}\right)$. From the SPGL1 dual solution, we have an estimate of $\lambda=\lambda\left(\epsilon_{0}\right)$. As noted above, this equivalence may not be very accurate, so the second step is to compute $\epsilon_{1}=\epsilon(\lambda)$ via FISTA, using a very high accuracy of $\delta=10^{-14}$. The pair $\left(\lambda, \epsilon_{1}\right)$ now leads to nearly equivalent solutions of $\left(\mathrm{QP}_{\lambda}\right)$ and $\left(\mathrm{BP}_{\epsilon}\right)$. The solution from FISTA will also be used to judge the accuracy of the other algorithms.

\footnotetext{
${ }^{7}$ Standard texts explain that $\left(\mathrm{BP}_{\epsilon}\right)$ is equivalent to minimizing $\|x\|_{\ell_{1}}+\chi_{\mathcal{Q}_{p}}(x)$, where $\mathcal{Q}_{p}$ is the feasible set $\left\{x:\|A x-b\|_{\ell_{2}} \leq \epsilon\right\}$ and $\chi_{\mathcal{Q}_{p}}(x)=0$ if $x \in \mathcal{Q}_{p}$ and $+\infty$ otherwise. Hence, the unconstrained problem has a discontinuous objective functional.
} 
The other main difficulty in comparisons is a fair stopping criterion. Each algorithm has its own stopping criterion (or may offer a choice of stopping criteria), and these are not directly comparable. To overcome this difficulty, we have modified the codes of the algorithms to allow for two new stopping criterion that we feel are the only fair choices. The short story is that we use NESTA to compute a solution $x_{N}$ and then ask the other algorithms to compute a solution that is at least as accurate.

Specifically, given NESTA's solution $x_{N}$ (using continuation), the other algorithms terminate at iteration $k$ when the solution $\hat{x}_{k}$ satisfies

$$
\text { (Crit. 1) }\left\|\hat{x}_{k}\right\|_{\ell_{1}} \leq\left\|x_{N}\right\|_{\ell_{1}} \text { and }\left\|b-A \hat{x}_{k}\right\|_{\ell_{2}} \leq 1.05\left\|b-A x_{N}\right\|_{\ell_{2}}
$$

or

$$
\text { (Crit. 2) } \quad \lambda\left\|\hat{x}_{k}\right\|_{\ell_{1}}+\frac{1}{2}\left\|A \hat{x}_{k}-b\right\|_{\ell_{2}}^{2} \leq \lambda\left\|x_{N}\right\|_{\ell_{1}}+\frac{1}{2}\left\|A x_{N}-b\right\|_{\ell_{2}}^{2} .
$$

We run tests with both stopping criteria to reduce any potential bias from the fact that some algorithms solve $\left(\mathrm{QP}_{\lambda}\right)$, for which Crit. 2 is the more natural, while others solve $\left(\mathrm{BP}_{\epsilon}\right)$, for which Crit. 1 is the more natural. In practice, the results when applying Crit. 1 or Crit. 2 are not significantly different.

\subsection{Numerical results.}

5.4.1. The case of exactly sparse signals. This first series of experiments tests all the algorithms discussed above in the case where the unknown signal is $s$-sparse with $s=m / 5$, $m=n / 8$, and $n=262,144$. This situation is close to the limit of perfect recovery from noiseless data. The $s$ nonzero entries of the signals $x^{0}$ are generated as described in (3.15). Reconstruction is performed with several values of the dynamic range $d=20,40,60,80,100$ in $\mathrm{dB}$. The measurement operator is a randomly subsampled DCT, as in section 4.1 (with a different random set of measurements chosen for each trial). The noise level is set to $\sigma=0.1$. The results are reported in Tables 3 (Crit. 1) and 4 (Crit. 2); each cell in these tables contains the mean value of $\mathcal{N}_{A}$ (the number of calls of $A$ or $A^{*}$ ) over 10 random trials, and, in a smaller font, the minimum and the maximum value of $\mathcal{N}_{A}$ over the 10 trials. When convergence is not reached after $\mathcal{N}_{A}=20,000$, we report DNC. As expected, the number of calls needed to reach convergence varies a lot from one algorithm to another.

The careful reader will notice that Tables 3 and 4 do not feature the results provided by 11_ls; indeed, while it seems faster than other interior point methods, it is still far from being comparable to the other algorithms reviewed here. In these experiments 11_ls typically needed 1,500 calls to $A$ or $A^{*}$ for reconstructing a $20 \mathrm{~dB}$ signal with $s=m / 100$ nonzero entries. For solving the same problem with a dynamic range of $100 \mathrm{~dB}$, it took about 5 hours to converge on a dual core MacPro G5 clocked at $2.7 \mathrm{GHz}$.

GPSR performs well in the case of low dynamic range signals; its performance, however, decreases dramatically as the dynamic range increases. Table 4 shows that it does not converge for 80 and $100 \mathrm{~dB}$ signals. GPSR with continuation does worse on the low dynamic range signals (which is not surprising). It does much better than the regular GPSR version on the high dynamic range signals, though it is slower than NESTA with continuation by more than a factor of 10. SpaRSA performs well at low dynamic range, comparable to NESTA, and begins to outperform GPSR with continuation as the dynamic range increases, although it

Copyright (c) by SIAM. Unauthorized reproduction of this article is prohibited. 
Table 3

Number of function calls $\mathcal{N}_{A}$ averaged over 10 independent runs. The sparsity level $s=m / 5$, and the stopping rule is Crit. 1 (5.1).

\begin{tabular}{l||c|c|c|c|c}
\hline Method & $20 \mathrm{~dB}$ & $40 \mathrm{~dB}$ & $60 \mathrm{~dB}$ & $80 \mathrm{~dB}$ & $100 \mathrm{~dB}$ \\
\hline NESTA & $446351 / 491$ & $880719 / 951$ & $17011581 / 1777$ & $45284031 / 4749$ & $146477729 / 15991$ \\
NESTA + Ct & $479475 / 485$ & $551539 / 559$ & $605589 / 619$ & $658635 / 679$ & $685657 / 705$ \\
GPSR & $5644 / 62$ & $733680 / 788$ & $53204818 / 5628$ & DNC & DNC \\
GPSR + Ct & $305293 / 311$ & $251245 / 257$ & $497453 / 531$ & $18161303 / 2069$ & $91017221 / 10761$ \\
SpaRSA & $345327 / 373$ & $455435 / 469$ & $542511 / 579$ & $601563 / 629$ & $708667 / 819$ \\
SPGL1 & $5437 / 61$ & $128102 / 142$ & $209190 / 216$ & $354297 / 561$ & $465380 / 562$ \\
FISTA & $6866 / 69$ & $270261 / 279$ & $935885 / 969$ & $34102961 / 3594$ & $1316411961 / 13911$ \\
FPC-AS & $156111 / 177$ & $236157 / 263$ & $218215 / 239$ & $351247 / 457$ & $325313 / 335$ \\
FPC-AS (CG) & $312212 / 359$ & $475301 / 538$ & $434423 / 481$ & $641470 / 812$ & $583567 / 595$ \\
FPC & $414394 / 436$ & $417408 / 422$ & $571546 / 594$ & $945852 / 1038$ & $39452018 / 4734$ \\
FPC-BB & $148140 / 152$ & $166158 / 168$ & $219208 / 250$ & $264252 / 282$ & $520320 / 800$ \\
Bregman-BB & $211203 / 225$ & $270257 / 295$ & $364355 / 393$ & $470429 / 501$ & $572521 / 657$ \\
\hline
\end{tabular}

Table 4

Number of function calls $\mathcal{N}_{A}$ averaged over 10 independent runs. The sparsity level $s=m / 5$, and the stopping rule is Crit. 2 (5.2).

\begin{tabular}{l||c|c|c|c|c}
\hline Method & $20 \mathrm{~dB}$ & $40 \mathrm{~dB}$ & $60 \mathrm{~dB}$ & $80 \mathrm{~dB}$ & $100 \mathrm{~dB}$ \\
\hline NESTA & $446351 / 491$ & $880719 / 951$ & $17011581 / 1777$ & $45284031 / 4749$ & $146477729 / 15991$ \\
NESTA + Ct & $479475 / 485$ & $551539 / 559$ & $605589 / 619$ & $658635 / 679$ & $685657 / 705$ \\
GPSR & $5944 / 64$ & $736678 / 790$ & $53164814 / 5630$ & $\mathrm{DNC}$ & $\mathrm{DNC}$ \\
GPSR +Ct & $305293 / 311$ & $251245 / 257$ & $511467 / 543$ & $18371323 / 2091$ & $91277251 / 10789$ \\
SpaRSA & $345327 / 373$ & $455435 / 469$ & $541509 / 579$ & $600561 / 629$ & $706667 / 819$ \\
SPGL1 & $5537 / 61$ & $138113 / 152$ & $217196 / 233$ & $358300 / 576$ & $470383 / 568$ \\
FISTA & $6563 / 66$ & $288279 / 297$ & $932882 / 966$ & $34072961 / 3591$ & $1316011955 / 13908$ \\
FPC-AS & $176169 / 183$ & $236157 / 263$ & $218215 / 239$ & $344247 / 459$ & $330319 / 339$ \\
FPC-AS (CG) & $357343 / 371$ & $475301 / 538$ & $434423 / 481$ & $622435 / 814$ & $588573 / 599$ \\
FPC & $416398 / 438$ & $435418 / 446$ & $577558 / 600$ & $899788 / 962$ & $38661938 / 4648$ \\
FPC-BB & $149140 / 154$ & $172164 / 174$ & $217208 / 254$ & $262248 / 286$ & $512308 / 790$ \\
Bregman-BB & $211203 / 225$ & $270257 / 295$ & $364355 / 393$ & $470429 / 501$ & $572521 / 657$ \\
\hline
\end{tabular}

begins to underperform NESTA with continuation in this regime. SpaRSA takes over twice as many function calls on the $100 \mathrm{~dB}$ signal as on the $20 \mathrm{~dB}$ signal.

SPGL1 shows good performance with very sparse signals and low dynamic range. Although it has fewer iteration counts than NESTA, the performance decreases much more quickly than for NESTA as the dynamic range increases; SPGL1 requires about $9 \times$ more calls to $A$ at $100 \mathrm{~dB}$ than at $20 \mathrm{~dB}$, whereas NESTA with continuation requires only about $1.5 \times$ more calls. FISTA is almost as fast as SPGL1 on the low dynamic range signal, but degrades very quickly as the dynamic range increases, taking about $200 \times$ more iterations at $100 \mathrm{~dB}$ than at $20 \mathrm{~dB}$. One large contributing factor to this poor performance at high dynamic range is the lack of a continuation scheme.

FPC performs well at low dynamic range, but is very slow on $100 \mathrm{~dB}$ signals. The BarzilaiBorwein version is consistently faster than the regular version, but also degrades much faster than NESTA with continuation as the dynamic range increases. Both FPC-AS and the Bregman algorithm perform well at all dynamic ranges, but again, degrade faster than NESTA

Copyright (c) by SIAM. Unauthorized reproduction of this article is prohibited. 


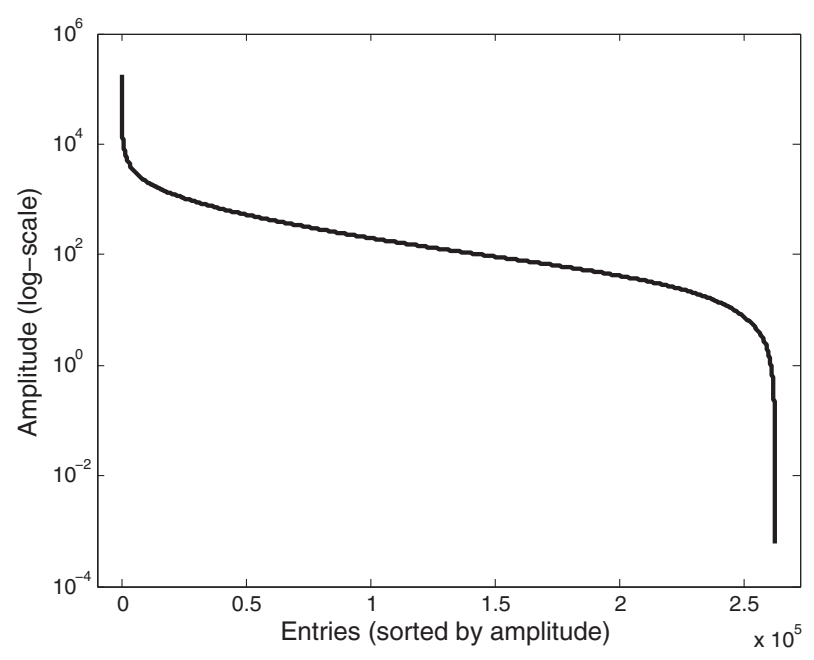

Figure 6. Sorted wavelet coefficients of the natural image used in the experiments.

with continuation as the dynamic range increases. There is a slight difference between the two FPC-AS versions (using L-BFGS or CG), but the dependence on the dynamic range is roughly similar.

The performances of NESTA with continuation are reasonable when the dynamic range is low. When the dynamic range increases, continuation becomes much more helpful. For some $100 \mathrm{~dB}$ signals, using continuation reduces the number of calls by a factor of 20 . In these experiments, the tolerance $\delta$ is consistently equal to $10^{-7}$; while this choice is reasonable when the dynamic range is high, it seems too conservative in the low dynamic range case. Setting a lower value of $\delta$ should improve NESTA's performance in this regime. In other words, NESTA with continuation might be tweaked to run faster on the low dynamic range signals. However, this is not in the spirit of this paper, and this is why we have not researched further refinements.

In summary, for exactly sparse signals exhibiting a significant dynamic range, (1) the performance of NESTA with continuation - but otherwise applied out-of-the-box - is comparable to that of state-of-the-art algorithms, and (2) most state-of-the-art algorithms are efficient on these types of signals.

5.4.2. Approximately sparse signals. We now turn our attention to approximately sparse signals. Such signals are generated via a permutation of the Haar wavelet coefficients of a $512 \times 512$ natural image. The data $b$ are $m=n / 8=32,768$ discrete cosine measurements selected at random. White Gaussian noise with standard deviation $\sigma=0.1$ is then added. Each test is repeated 5 times, using a different random permutation every time (as well as a new instance of the noise vector). Unlike in the exactly sparse case, the wavelet coefficients of natural images contain mostly mid-range and low level coefficients (see Figure 6) which are challenging to recover.

The results are reported in Tables 5 (Crit. 1) and 6 (Crit. 2); the results from applying the two stopping criteria are nearly identical. In this series of experiments, the performance of SPGL1 is quite good, but seems to vary a lot from one trial to another (Table 6). Notice that 
Table 5

Recovery results of an approximately sparse signal with Crit. 1 as a stopping rule.

\begin{tabular}{l||c|c|c}
\hline Method & $\left\langle\mathcal{N}_{A}\right\rangle$ & $\min \mathcal{N}_{A}$ & $\max \mathcal{N}_{A}$ \\
\hline NESTA & 18912 & 18773 & 19115 \\
NESTA + Ct & 2667 & 2603 & 2713 \\
GPSR & DNC & DNC & DNC \\
GPSR + Ct & DNC & DNC & DNC \\
SpaRSA & 10019 & 8369 & 12409 \\
SPGL1 & 1776 & 1073 & 2464 \\
FISTA & 10765 & 10239 & 11019 \\
FPC-AS & DNC & DNC & DNC \\
FPC-AS (CG) & DNC & DNC & DNC \\
FPC & DNC & DNC & DNC \\
FPC-BB & DNC & DNC & DNC \\
Bregman-BB & 2045 & 2045 & 2045 \\
\hline
\end{tabular}

Table 6

Recovery results of an approximately sparse signal with Crit. 2 as a stopping rule.

\begin{tabular}{l||c|c|c}
\hline Method & $\left\langle\mathcal{N}_{A}\right\rangle$ & $\min \mathcal{N}_{A}$ & $\max \mathcal{N}_{A}$ \\
\hline NESTA & 18912 & 18773 & 19115 \\
NESTA +Ct & 2667 & 2603 & 2713 \\
GPSR & DNC & DNC & DNC \\
GPSR + Ct & DNC & DNC & DNC \\
SpaRSA & 10021 & 8353 & 12439 \\
SPGL1 & 1776 & 1073 & 2464 \\
FISTA & 10724 & 10197 & 10980 \\
FPC-AS & DNC & DNC & DNC \\
FPC-AS (CG) & DNC & DNC & DNC \\
FPC & DNC & DNC & DNC \\
FPC-BB & DNC & DNC & DNC \\
Bregman-BB & 2045 & 2045 & 2045 \\
\hline
\end{tabular}

the concept of an active set is ill defined in the approximately sparse case; as a consequence, the active set version of FPC is not much of an improvement over the regular FPC version. FPC is very fast for $s$-sparse signals, but lacks the robustness to deal with less ideal situations in which the unknown is only approximately sparse.

FISTA and SpaRSA converge for these tests, but are not competitive with the best methods. It is reasonable to assume that FISTA would also improve if implemented with continuation. SpaRSA already uses continuation, but does not match its excellent performance on exactly sparse signals.

Bregman, SPGL1, and NESTA with continuation all have excellent performances (continuation really helps NESTA) in this series of experiments. NESTA with continuation seems very robust when high accuracy is required. The main distinguishing feature of NESTA is that it is less sensitive to dynamic range; this means that as the dynamic range increases, or as the noise level $\sigma$ decreases, NESTA becomes very competitive. For example, when the same test was repeated with more noise $(\sigma=1)$, all the algorithms converged faster. In moving from $\sigma=1$ to $\sigma=0.1$, SPGL1 required 90\% more iterations and Bregman required $20 \%$ more iterations, while NESTA with continuation required only $5 \%$ more iterations.

Copyright (c) by SIAM. Unauthorized reproduction of this article is prohibited. 
One conclusion from these tests is that SPGL1, Bregman, and NESTA (with continuation) are the only methods that effectively deal with approximately sparse signals. The other methods, most of which did very well on exactly sparse signals, take more than 10,000 function calls or even do not converge in 20,000 function calls; by comparison, SPGL1, Bregman, and NESTA with continuation converge in about 2,000 function calls. It is also worth noting that Bregman is only as good as the subproblem solver; though not reported here, using the regular FPC (instead of FPC-BB) with Bregman leads to much worse performance.

The algorithms which did converge all achieved a mean relative $\ell_{1}$ error (using (4.1) and the high accuracy FISTA solution as the reference) less than $2 \cdot 10^{-4}$ and sometimes as low as $10^{-5}$, except SPGL1, which had a mean relative error of $1.1 \cdot 10^{-3}$. Of the algorithms that did not converge in 20,000 function calls, FPC and FPC-BB had a mean $\ell_{1}$ relative error of about $5 \cdot 10^{-3}$, GPSR with continuation had errors of about $5 \cdot 10^{-2}$, and the rest had errors greater than $10^{-1}$.

6. An all-purpose algorithm. A distinguishing feature is that NESTA is able to cope with a wide range of standard regularizing functions. In this section, we present two examples: nonstandard $\ell_{1}$ minimization and TV minimization.

6.1. Nonstandard sparse reconstruction: $\ell_{1}$ analysis. Suppose we have a signal $x \in \mathbb{R}^{n}$, which is assumed to be approximately sparse in a transformed domain such as the wavelet, the curvelet, or the time-frequency domains. Let $W$ be the corresponding synthesis operator whose columns are the waveforms we use to synthesize the signal $x=W \alpha$ (real-world signals do not admit an exactly sparse expansion); e.g., the columns may be wavelets, curvelets, or any dictionary (or combination of dictionaries). We will refer to $W^{*}$ as the analysis operator. As before, we have (possibly noisy) measurements $b=A x^{0}+z$. The synthesis approach attempts reconstruction by solving

$$
\begin{array}{ll}
\text { minimize } & \|\alpha\|_{\ell_{1}} \\
\text { subject to } & \|b-A W \alpha\|_{\ell_{2}} \leq \epsilon,
\end{array}
$$

while the analysis approach solves the related problem

$$
\begin{array}{ll}
\text { minimize } & \left\|W^{*} x\right\|_{\ell_{1}} \\
\text { subject to } & \|b-A x\|_{\ell_{2}} \leq \epsilon .
\end{array}
$$

If $W$ is orthonormal, the two problems are equivalent, but, in general, these approaches give distinct solutions, and current theory explaining the differences is still in its infancy. The article [31] suggests that synthesis may be overly sensitive and argues with geometric heuristics and numerical simulations that analysis is sometimes preferable.

Solving $\ell_{1}$-analysis problems with NESTA is straightforward, as only step 1 need be adapted. We have

$$
f_{\mu}(x)=\max _{u \in \mathcal{Q}_{p}}\left\langle u, W^{*} x\right\rangle-\frac{\mu}{2}\|u\|_{\ell_{2}}^{2},
$$

and the gradient at $x$ is equal to

$$
\nabla f_{\mu}(x)=W u_{\mu}(x)
$$

here, $u_{\mu}(x)$ is given by

Copyright (c) by SIAM. Unauthorized reproduction of this article is prohibited. 


$$
\left(u_{\mu}(x)\right)[i]= \begin{cases}\mu^{-1}\left(W^{*} x\right)[i] & \text { if }\left|\left(W^{*} x\right)[i]\right|<\mu, \\ \operatorname{sgn}\left(\left(W^{*} x\right)[i]\right) & \text { otherwise. }\end{cases}
$$

Steps 2 and 3 remain unchanged. The computational complexity of the algorithm is then increased by an extra term, namely $2 \mathcal{C}_{W}$, where $\mathcal{C}_{W}$ is the cost of applying $W$ or $W^{*}$ to a vector. In practical situations, there is often a fast algorithm for applying $W$ and $W^{*}$, e.g., a fast wavelet transform [43], a fast curvelet transform [11], or a fast short-time Fourier transform [43], which makes this a low-cost extra step. ${ }^{8}$

6.2. Numerical results for nonstandard $\ell_{1}$ minimization. Because NESTA is one of very few algorithms that can solve efficiently both the analysis and synthesis problems, we tested the performance of both analysis and synthesis on a simulated real-world signal from the field of radar detection. The test input is a superposition of three signals. The first signal, which is intended to make recovery more difficult for any smaller signals, is a plain sinusoid with amplitude of 1,000 and frequency near $835 \mathrm{MHz}$.

A second signal, similar to a Doppler pulse radar, is at a carrier frequency of $2.33 \mathrm{GHz}$ with a maximum amplitude of 10 , a pulse width of $1 \mu \mathrm{s}$, and a pulse repetition interval of $10 \mu \mathrm{s}$; the pulse envelope is trapezoidal, with a $10 \mathrm{~ns}$ rise time and $40 \mathrm{~ns}$ fall time. This signal is more than $40 \mathrm{~dB}$ lower than the pure sinusoid, since the maximum amplitude is $100 \times$ smaller and the radar is nonzero only $10 \%$ of the time. The Doppler pulse was chosen to be roughly similar to a realistic weather Doppler radar. In practice, these systems operate at $5 \mathrm{~cm}$ or $10 \mathrm{~cm}$ wavelengths (i.e., 6 or $3 \mathrm{GHz}$ ) and send out short trapezoidal pulses to measure the radial velocity of water droplets in the atmosphere using the Doppler effect.

The third signal, which is the signal of interest, is a frequency-hopping radar pulse with maximum amplitude of 1 (so about $20 \mathrm{~dB}$ beneath the Doppler signal and more than $60 \mathrm{~dB}$ below the sinusoid). For each instance of the pulse, the frequency is chosen uniformly at random from the range $200 \mathrm{MHz}$ to $2.4 \mathrm{GHz}$. The pulse duration is $2 \mu$ s and the pulse repetition interval is $22 \mu \mathrm{s}$, which means that some, but not all, pulses overlap with the Doppler radar pulses. The rise time and fall time of the pulse envelope are comparable to those of the Doppler pulse. Frequency-hopping signals may arise in applications because they can be more robust to interference and because they can be harder to intercept. When the carrier frequencies are not known to the listener, the receiver must be designed to cover the entire range of possible frequencies $(2.2 \mathrm{GHz}$ in our case). While some current analog-todigital converters (ADCs) may be capable of operating at $2.2 \mathrm{GHz}$, they do so at the expense of low precision. Hence this situation may be particularly amenable to a compressed sensing setup by using several slower (but accurate) ADCs to cover a large bandwidth.

We consider the exact signal to be the result of an infinite-precision ADC operating at $5 \mathrm{GHz}$, which corresponds to the Nyquist rate for signals with $2.5 \mathrm{GHz}$ of bandwidth. Measurements are taken using an orthogonal Hadamard transform with randomly permuted columns, and these measurements are subsequently subsampled by randomly choosing $m=.3 n$ rows of the transform (so that we undersample Nyquist by 10/3). Samples are recorded for $T=209.7 \mu \mathrm{s}$, which corresponds to $n=2^{20}$. White noise is added to the measurements

\footnotetext{
${ }^{8}$ The ability to solve the analysis problem also means that NESTA can easily solve reweighted $\ell_{1}$ problems [16] with no change to the code.
} 
to make a $60 \mathrm{~dB}$ signal-to-noise ratio (SNR) (note that the effective SNR for the frequencyhopping pulse is much lower). The frequencies of the sinusoid and the Doppler radar are chosen such that they were not integer multiples of the lowest recoverable frequency $f_{\min }=1 /(2 T)$.

For reconstruction, the signal is analyzed with a tight frame of Gabor atoms that is approximately $5.5 \times$ overcomplete. The particular parameters of the frame are chosen to give reasonable reconstruction, but were not tweaked excessively. It is likely that differences in performance between analysis and synthesis are heavily dependent on the particular dictionary.

To analyze performance, we restrict our attention to the frequency domain in order to simplify comparisons. The top plot in Figure 7 shows the frequency components of the original, noiseless signal. The frequency-hopping pulse barely shows up since the amplitude is $1,000 \times$ smaller than the sinusoid and since each frequency occurs only for $1 \mu \mathrm{s}$ (of $210 \mu \mathrm{s}$ total).

The middle and bottom plots in Figure 7 show the spectrum of the recovered signal using analysis and synthesis, respectively. For this test, analysis does a better job of finding the frequencies belonging to the small pulse, while synthesis does a better job of recreating the large pulse and the pure tone. The two reconstructions used slightly different values of $\mu$ to account for the redundancy in the size of the dictionary; otherwise, algorithm parameters were the same. In the analysis problem, NESTA took 231 calls to the analysis/synthesis operator (and 231 calls to the Hadamard transform); for synthesis, NESTA took 1,378 calls to the analysis/synthesis operator (and 1,378 to the Hadamard transform). With NESTA, synthesis is more computationally expensive than analysis since no change-of-variables trick can be done; in the synthesis case, $W$ and $W^{*}$ are used in steps 2 and 3, while in the analysis case, the same operators are used once in step 1 (this is accomplished by the previously mentioned change of variables for partial orthogonal measurements).

As emphasized in [31], when $W$ is overcomplete, the solution computed by solving the analysis problems is likely to be denser than in the synthesis case. In plain English, the analysis solution may seem "noisier" than the synthesis solution. But the compactness of the solution of the synthesis problem may also be its weakness: an error on one entry of $\alpha$ may lead to a solution that differs a lot. This may explain why the frequency-hopping radar pulse is harder to recover with the synthesis prior.

Because all other known first-order methods solve only the synthesis problem, NESTA may prove to be extremely useful for real-world applications. Indeed, this simple test suggests that analysis may sometimes be much preferable to synthesis, and, given a signal with $2^{20}$ samples (too large for interior point methods), we know of no other algorithm that can return the same results.

6.3. TV minimization. Nesterov's framework also makes TV minimization possible. The TV norm of a two-dimensional digital object $x[i, j]$ is given by

$$
\|x\|_{T V}:=\sum_{i, j}\|\nabla x[i, j]\|, \quad \nabla x[i, j]=\left[\begin{array}{l}
\left(D_{1} x\right)[i, j] \\
\left(D_{2} x\right)[i, j]
\end{array}\right],
$$

where $D_{1}$ and $D_{2}$ are the horizontal and vertical differences

$$
\begin{aligned}
& \left(D_{1} x\right)[i, j]=x[i+1, j]-x[i, j], \\
& \left(D_{2} x\right)[i, j]=x[i, j+1]-x[i, j] .
\end{aligned}
$$

Copyright $\odot$ by SIAM. Unauthorized reproduction of this article is prohibited. 

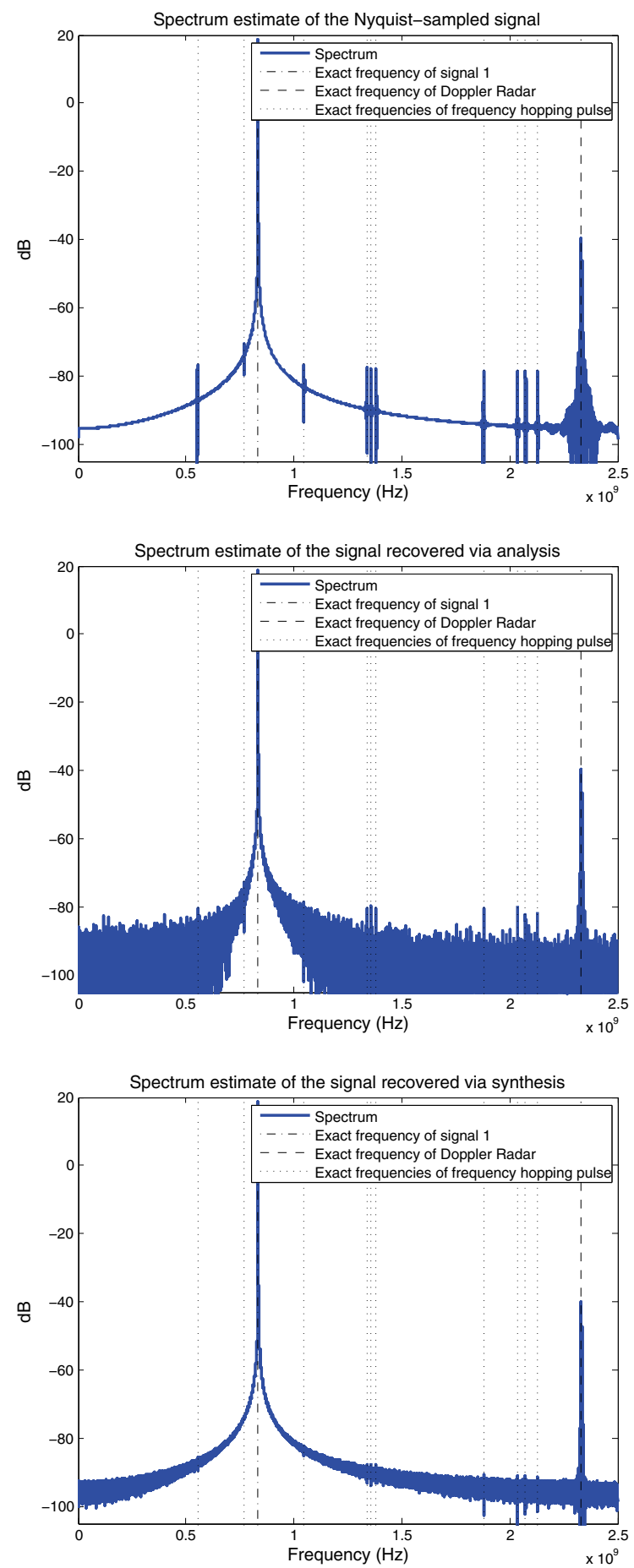

Figure 7. Top: spectrum estimate of the exact signal, no noise. The pure tone at $60 \mathrm{~dB}$ and the Doppler radar at $20 d B$ dominate the $0 d B$ frequency-hopping pulses. Middle: spectrum estimate of the recovered signal using analysis prior, with $60 \mathrm{~dB}$ SNR. The spectrum appears noisy, but the frequency-hopping pulses stand out. Bottom: spectrum estimate of the recovered signal using synthesis prior, with $60 \mathrm{~dB}$ SNR. The spectrum appears cleaner, but the small $0 \mathrm{~dB}$ pulses do not appear.

Copyright $@$ by SIAM. Unauthorized reproduction of this article is prohibited. 
Now the TV norm can be expressed as follows:

$$
\|x\|_{T V}=\max _{u \in \mathcal{Q}_{d}}\langle u, D x\rangle
$$

where $u=\left[u_{1}, u_{2}\right]^{*} \in \mathcal{Q}_{d}$ if and only for each $(i, j), u_{1}^{2}[i, j]+u_{2}^{2}[i, j] \leq 1$ and $D=\left[D_{1}, D_{2}\right]^{*}$. The key feature of Nesterov's work is to smooth a well-structured nonsmooth function as follows (notice in (6.3) the similarity between the TV norm and the $\ell_{1}$ norm):

$$
\max _{u \in \mathcal{Q}_{d}}\langle u, D x\rangle-\mu p_{d}(u)
$$

Choosing $p_{d}(u)=\frac{1}{2}\|u\|_{\ell_{2}}^{2}$ provides a reasonable prox-function that eases the computation of $\nabla f_{\mu}$. Just as before, changing the regularizing function modifies only step 1 of NESTA. Here,

$$
f_{\mu}(x)=\max _{u \in \mathcal{Q}_{d}}\langle u, D x\rangle-\frac{\mu}{2}\|u\|_{\ell_{2}}^{2} .
$$

Then, as usual,

$$
\nabla f_{\mu}(x)=D^{*} u^{(\mu)}(x),
$$

where $u^{(\mu)}(x)$ is of the form $\left[u_{1}^{(\mu)}, u_{2}^{(\mu)}\right]^{*}$ and, for each $a \in\{1,2\}$,

$$
u_{a}^{(\mu)}[i, j]= \begin{cases}\mu^{-1}\left(D_{a} x\right)[i, j] & \text { if }\|\nabla x[i, j]\|<\mu, \\ \|\nabla x[i, j]\|^{-1}\left(D_{a} x\right)[i, j] & \text { otherwise. }\end{cases}
$$

The application of $D$ and $D^{*}$ leads to a negligible computational cost (sparse matrix-vector multiplications).

6.4. Numerical results for TV minimization. We are interested in solving

$$
\begin{array}{ll}
\text { minimize } & \|x\|_{T V} \\
\text { subject to } & \|b-A x\|_{\ell_{2}} \leq \epsilon .
\end{array}
$$

To be sure, a number of efficient TV minimization algorithms have been proposed to solve (6.4) in the special case $A=I$ (denoising problem); see [17, 22, 35, 61]. In comparison, only a few methods have been proposed to solve the more general problem (6.4) even when $A$ is a projector. Known methods include interior point methods $\left(\ell_{1}\right.$-magic) [10], proximalsubgradient methods [5, 19], split Bregman [35], and the very recently introduced RecPF ${ }^{9}$ [59], which operates in the special case of partial Fourier measurements. Applications of some of Nesterov's works have also been considered in different settings [56, 21, 2].

Again, we compare NESTA with algorithms with publicly available implementations. Therefore, the two algorithms we tested are RecPF (the newest member of the family) and TwIST [5]. ( $\ell_{1}$-magic is based on an interior point method and is not yet applicable to this large-scale problem.)

Evaluations are made by comparing the performances of NESTA (with continuation), RecPF, and TwIST on a set of images composed of random squares. As in section 5, the dynamic range of the signals (amplitude of the squares) varies in a range of $20-40 \mathrm{~dB}$. The

\footnotetext{
${ }^{9}$ Code is available at http://www.caam.rice.edu/ optimization/L1/RecPF/.
} 


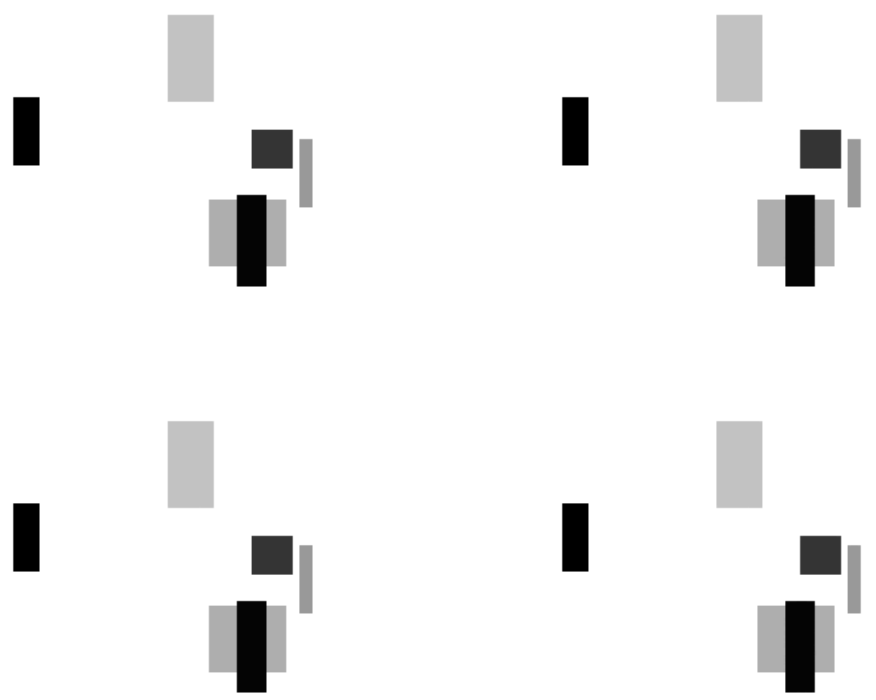

Figure 8. Top left: original image of size $1024 \times 1024$ with a dynamic range of about 40 dB. Top right: RecPF solution. Bottom left: TwIST solution. Bottom right: NESTA solution.

size of each image $x$ is $1,024 \times 1,024$; one of these images is displayed in the top panel of Figure 8. The data $b$ are partial Fourier measurements as in [13]; the number of measurements $m=n / 10$. White Gaussian noise of standard deviation $\sigma=0.1$ is added. The parameters of NESTA are set up as follows:

$$
x_{0}=A^{*} b, \quad \mu=0.2, \quad \delta=10^{-5}, \quad T=5,
$$

and the initial value of $\mu$ is

$$
\mu_{0}=0.9 \max _{i j}\left\|\nabla x_{0}[i, j]\right\|
$$

The maximal number of iterations is set to $\mathcal{I}_{\max }=4,000$. As it turns out, TV minimization from partial Fourier measurements is of significant interest in the field of magnetic resonance imaging [42].

As discussed above, RecPF has been designed to solve TV minimization reconstruction problems from partial Fourier measurements. We set the parameters of RecPF to their default values except for the parameter tol_rel_inn, which is set to $10^{-5}$. TwIST calls Chambolle's algorithm [17] at each iteration to compute the proximity operator of TV; the maximum number of iterations in Chambolle's algorithm is set to 10. With these parameter selections, TwIST and RecPF converge to a solution close enough to NESTA's output - at least in our experiments. Figure 8 shows the solution computed by RecPF (top right), TwIST (bottom left), and NESTA (bottom left).

The curves in Figure 9 show the number of calls to $A$ or $A^{*}$; midpoints are averages over 5 random trials, with error bars indicating the minimum and maximum numbers of calls. 


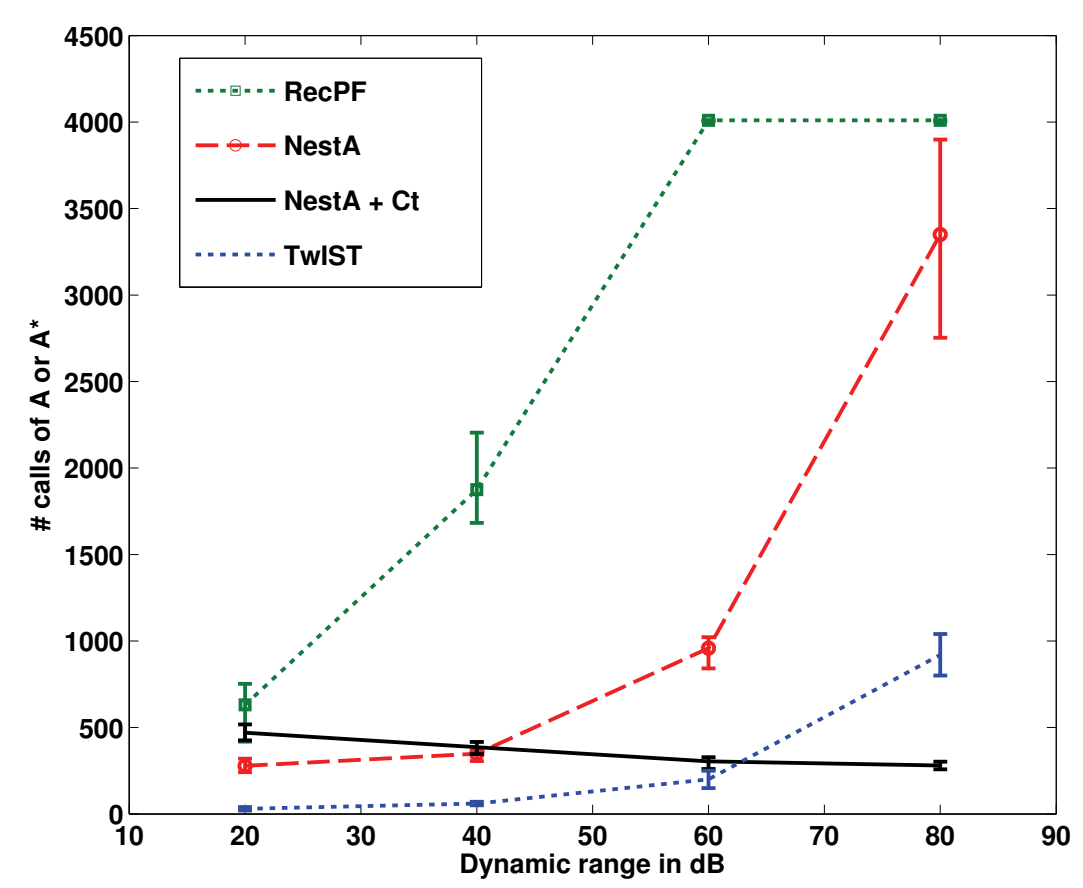

Figure 9. Number of calls to $A$ and $A^{*}$ as a function of the dynamic range. Solid line: NESTA with continuation. Dashed line: NESTA. Upper dotted line: RecPF. In the 60 and $80 \mathrm{~dB}$ experiments, RecPF did not converge to the solution in the allowed number of iterations (4,000), and this is the reason the number of calls saturates. Lower dotted line: TwIST. For TwIST, it is important not to take the number of function calls to $A$ and $A^{*}$ as a proxy for the computational time of the algorithm. Indeed, the dominant computational cost in TwIST is in evaluating the proximity operator; see Table 7.

Here, RecPF is stopped when

$$
\begin{aligned}
\left\|x_{\operatorname{RecPF}}\right\|_{T V} & \leq 1.05\left\|x_{N}\right\|_{T V}, \\
\left\|b-A x_{\operatorname{RecPF}}\right\|_{\ell_{2}} & \leq 1.05\left\|b-A x_{N}\right\|_{\ell_{2}},
\end{aligned}
$$

where $x_{N}$ is the solution computed via NESTA. As before, continuation is very efficient when the dynamic range is high (typically higher than $40 \mathrm{~dB}$ ). An interesting feature is that the numbers of calls are very similar over all 5 trials. When the dynamic range increases, the computational costs of both NESTA and RecPF naturally increase. Note that in the 60 and $80 \mathrm{~dB}$ experiments, RecPF did not converge to the solution, and this is the reason the number of calls saturates. While both methods have a similar computational cost in the low dynamic range regime, NESTA has a clear advantage in the higher dynamic range regime. Moreover, the number of iterations needed to reach convergence with NESTA with continuation is fairly low-300-400 calls to $A$ and $A^{*}$ - and so this algorithm is well suited to large-scale problems.

Figure 9 suggests that TwIST performs well, in terms of function calls, on the problems with low dynamic range, but deteriorates as the dynamic range increases. However, this hides a fundamental complication of the TwIST algorithm: the function calls to $A$ and $A^{*}$ are not always the dominant computational cost. TwIST is one of many TV methods that is a proximal gradient algorithm, meaning that at each step, it relies on the solution to a proximity

Copyright (C) by SIAM. Unauthorized reproduction of this article is prohibited. 
Table 7

Comparison of computation times, mean value over 5 trials (in seconds).

\begin{tabular}{l||c|c|c|c}
\hline Method & $20 \mathrm{~dB}$ & $40 \mathrm{~dB}$ & $60 \mathrm{~dB}$ & $80 \mathrm{~dB}$ \\
\hline NESTA + Ct & 750 & 704 & 553 & 542 \\
RecPF & 725 & 1918 & DNC & DNC \\
TwIST & 203 & 553 & 1675 & 9510 \\
\hline
\end{tabular}

operator:

$$
\operatorname{Prox}_{T V, \gamma}(z)=\underset{x}{\operatorname{argmin}} \gamma\|x\|_{T V}+\frac{1}{2}\|x-z\|_{\ell_{2}}^{2}
$$

see [20] and the references therein. Evaluating the proximity operator at $z$ is equivalent to solving a TV denoising problem, which is far from trivial. ${ }^{10}$ In [5], the authors advocate the use of a side algorithm (for instance, Chambolle's algorithm [17]) to do this.

There are a few issues with this approach. The first is that side algorithms depend on various parameters, which adds complexity. The second is that these denoising algorithms are computationally demanding, which makes them hard to apply to large-scale problems. To illustrate this phenomenon, we compared the computation times of NESTA + Ct, RecPF, and TwIST on the same kind of synthetic $1,024 \times 1,024$ images. The results are reported in Table 7. Each line displays the computation time before convergence is reached. We observe that while TwIST needs fewer calls to $A$ or $A^{*}$ than NESTA when the dynamic range is low, the computational cost of each step is higher (and increases as the problem size increases) because the dominant computational cost in TwIST is in evaluating the proximity operator.

7. Discussion. In this paper, we have proposed an algorithm for general sparse recovery problems, which is based on Nesterov's method. This algorithm, NESTA, is accurate and competitive with state-of-the-art alternatives. In fact, in applications of greatest interest such as the recovery of approximately sparse signals, it outperforms most of the existing methods we have used in our comparisons and is comparable to the best. Furthermore, what is interesting here is that we have not attempted to optimize the algorithm in any way. For instance, we have not optimized the parameters $\left\{\alpha_{k}\right\}$ and $\left\{\tau_{k}\right\}$ or the number of continuation steps as a function of the desired accuracy $\delta$, and so it is expected that finer tuning would speed up the algorithm. Another advantage is that NESTA is extremely flexible in the sense that minor adaptations lead to efficient algorithms for a host of optimization problems that are crucial in the field of signal/image processing.

7.1. Extensions. This paper focuses on the situation in which $A^{*} A$ is a projector (the rows of $A$ are orthonormal). This stems from the facts that (1) the most computationally friendly versions of compressed sensing are of this form, and (2) this situation allows fast computations of the two sequences of iterates $\left\{y_{k}\right\}$ and $\left\{z_{k}\right\}$. It is important, however, to extend NESTA so as to be able to cope with a wider range of problems in which $A^{*} A$ is not a projection (or not diagonal).

\footnotetext{
${ }^{10}$ For $\ell_{1}$ minimization, the equivalent proximity operator is given by shrinkage and can be solved in linear time. In the case of the weighted $\ell_{1}$ problem, using $\|W x\|_{1}$ in the objective, the proximity operator is not known in closed form unless $W$ is diagonal. Since most first-order $\ell_{1}$ algorithms rely on the proximity operator, this is the reason that no other algorithms can solve the analysis problem, in contrast to NESTA.
}

Copyright (c) by SIAM. Unauthorized reproduction of this article is prohibited. 
In order to do this, observe that in steps 2 and 3, we need to solve problems of the form

$$
y_{k}=\underset{x \in \mathcal{Q}_{p}}{\operatorname{argmin}}\|x-q\|_{\ell_{2}}^{2}
$$

for some $q$, and we have seen that the solution is given by $y_{k}=\mathcal{P}_{\mathcal{Q}_{p}}(q)$, where $\mathcal{P}_{\mathcal{Q}_{p}}$ is the projector onto $\mathcal{Q}_{p}:=\left\{x:\|A x-b\|_{\ell_{2}} \leq \epsilon\right\}$. The solution is given by

$$
y_{k}=\left(I+\lambda A^{*} A\right)^{-1}\left(q+\lambda A^{*} b\right)
$$

for some $\lambda \geq 0$. When the eigenvalues of $A^{*} A$ are well clustered, the right-hand side of (7.1) can be computed very efficiently via a few CG steps. Note that this is of direct interest in compressed sensing applications in which $A$ is a random matrix since in all the cases we are familiar with, the eigenvalues of $A^{*} A$ are tightly clustered. Hence, NESTA may be extended to general problems while retaining its efficiency, with the proviso that a good rule for selecting $\lambda$ in (7.1) is available, i.e., such that $\left\|A y_{k}-b\right\|_{\ell_{2}}=\epsilon$ unless $q \in \mathcal{Q}_{p}$. Of course, one can always eliminate the problem of finding such a $\lambda$ by solving the unconstrained problem $\left(\mathrm{QP}_{\lambda}\right)$ instead of $\left(\mathrm{BP}_{\epsilon}\right)$. In this case, each NESTA iteration is actually very cheap, no matter what $A$ looks like.

It is worth noting that if $A$ is relatively small and such that it is computationally possible to perform a one-time singular value decomposition of $A$ (this may be the case if $A$ is not too large, but the analysis dictionary $W$ is very overcomplete and is the bottleneck for computation), then the inversion requires just two matrix multiplications. The correct value of $\lambda$ is given by a scalar equation that may be solved efficiently by Newton's method (see also [56] for a similar approach to selecting $\lambda$ ).

Finally, we also observe that Nesterov's framework is likely to provide efficient algorithms for related problems which do not have the special $\ell_{1}+\ell_{2}^{2}$ structure. One example might be the Dantzig selector, which is a convenient and flexible estimator for recovering sparse signals from noisy data [15]:

$$
\begin{array}{ll}
\text { minimize } & \|x\|_{\ell_{1}} \\
\text { subject to } & \left\|A^{*}(b-A x)\right\|_{\ell_{\infty}} \leq \delta .
\end{array}
$$

This is, of course, equivalent to the unconstrained problem

$$
\operatorname{minimize} \quad \lambda\|x\|_{\ell_{1}}+\left\|A^{*}(b-A x)\right\|_{\ell_{\infty}}
$$

for some value of $\lambda$. Clearly, one could apply Nesterov's smoothing techniques to smooth both terms in the objective functional together with Nesterov's accelerated gradient techniques, and derive a novel and efficient algorithm for computing the solution to the Dantzig selector. This is just one of many examples. Another might be the minimization of a sum of two norms, e.g., an $\ell_{1}$ and a TV norm, under data constraints.

7.2. Software. In the spirit of reproducible research [27], a MATLAB version of NESTA has been made available at http://www.acm.caltech.edu/ nesta/. 
Acknowledgments. The authors are grateful to the anonymous reviewers for helpful comments and for pointing out [30, 40, 41]. S. Becker wishes to thank Peter Stobbe for the use of his Hadamard transform and Gabor frame code, and Wotao Yin for helpful discussions about RecPF. J. Bobin wishes to thank Hamza Fawzi for fruitful discussions, and E. Candès would like to thank Jalal Fadili for his suggestions. We are grateful to Stephen Wright for his comments on an earlier version of this paper, for suggesting that we use a better version of GPSR, and for encouraging us to test SpaRSA. Thanks, Stephen!

\section{REFERENCES}

[1] M. V. Afonso, J. M. Biouchs-Dias, And M. A. T. Figueiredo, Fast image recovery using variable splitting and constrained optimization, IEEE Trans. Image Process., 19 (2010), pp. 2345-2356.

[2] J.-F. Aujol, Some first-order algorithms for total-variation based image restoration, J. Math. Imaging Vision, 34 (2009), pp. 307-327.

[3] J. Barzilai And J. Borwein, Two point step size gradient method, IMA J. Numer. Anal., 8 (1988), pp. $141-148$

[4] A. BeCK AND M. TeBoulle, Fast iterative shrinkage-thresholding algorithm for linear inverse problems, SIAM J. Imaging Sci., 2 (2009), pp. 183-202.

[5] J. M. Bioucas-Dias and M. A. T. Figueiredo, A new TwIST: Two-step iterative shrinkage/ thresholding algorithms for image restoration, IEEE Trans. Image Process., 16 (2007), pp. 2992-3004.

[6] E. G. Birgin, J. M. Martínez, and M. Raydan, Nonmonotone spectral projected gradient methods on convex sets, SIAM J. Optim., 10 (2000), pp. 1196-1211.

[7] J. Bobin AND E. J. CANDÈs, A fast and accurate first-order algorithm for compressed sensing, in Proceedings of the 16th IEEE International Conference on Image Processing (ICIP'09), 2009, pp. 1457-1460.

[8] J. Bobin, J.-L. Starck, And R. Ottensamer, Compressed sensing in astronomy, IEEE J. Sel. Top. Signal Process., 2 (2008), pp. 718-726.

[9] J.-F. Cai, S. Osher, And Z. Shen, Convergence of the linearized Bregman iterations for 1 1-norm minimization, Math. Comp., 78 (2009), pp. 2127-2136.

[10] E. J. CAndès, $\ell_{1}$-Magic, Technical report, Caltech, Pasadena, CA, 2007.

[11] E. Candès, L. Demanet, D. Donoho, and L. Ying, Fast discrete curvelet transforms, Multiscale Model. Simul., 5 (2006), pp. 861-899.

[12] E. J. CANDÈs And Y. Plan, Near-ideal model selection by $\ell_{1}$ minimization, Ann. Statist., 37 (2007), pp. $2145-2177$.

[13] E. J. Candès, J. Romberg, and T. TAO, Robust uncertainty principles: Exact signal reconstruction from highly incomplete frequency information, IEEE Trans. Inform. Theory, 52 (2006), pp. 489-509.

[14] E. J. CANDÈs AND T. TAO, Near optimal signal recovery from random projections: Universal encoding strategies?, IEEE Trans. Inform. Theory, 52 (2006), pp. 5406-5425.

[15] E. J. CANDÈs AND T. TAO, The Dantzig selector: Statistical estimation when $p$ is much larger than n, Ann. Statist., 35 (2007), pp. 2313-2351.

[16] E. J. CANDÈs, M. B. WAKin, AND S. P. Boyd, Enhancing sparsity by reweighted $\ell_{1}$ minimization, J. Fourier Anal. Appl., 14 (2008), pp. 877-905.

[17] A. Chambolle, An algorithm for total-variation minimization and applications, J. Math. Imaging Vision, 20 (2004), pp. 89-97.

[18] S. S. Chen, D. L. Donoho, and M. A. SAunders, Atomic decomposition by basis pursuit, SIAM J. Sci. Comput., 20 (1998), pp. 33-61.

[19] P. L. Combettes And J. C. Pesquet, A proximal decomposition method for solving convex variational inverse problems, Inverse Problems, 24 (2008), 065014.

[20] P. L. Combettes And V. R. Wajs, Signal recovery by proximal forward-backward splitting, Multiscale Model. Simul., 4 (2005), pp. 1168-1200.

[21] J. Dahl, C. Hansen, S. H. Jensen, And T. L. Jensen, Algorithms and software for total variation image reconstruction via first-order methods, Numer. Algorithms, 53 (2010), pp. 67-92.

Copyright (c) by SIAM. Unauthorized reproduction of this article is prohibited. 
[22] J. Darbon and M. Sigelle, A fast and exact algorithm for total-variation minimization, in Proceedings of the 2nd Iberian Conference on Pattern Recognition and Image Analysis (IbPRIA), Lecture Notes in Comput. Sci. 3522, Springer-Verlag, Berlin, 2005, pp. 351-359.

[23] DARPA, Analog-to-Information Receiver Development Program (A-to-I), DARPA Broad Agency Announcement BAA08-03, 2007; available online from http://www.darpa.mil/mto/Solicitations/ baa08-03/index.html.

[24] I. Daubechies, M. Defrise, And C. De Mol, An iterative thresholding algorithm for linear inverse problems with a sparsity constraint, Comm. Pure Appl. Math., 57 (2004), pp. 1413-1457.

[25] I. Daubechies, M. Fornasier, And I. Loris, Accelerated projected gradient method for linear inverse problems with sparsity constraints, J. Fourier Anal. Appl., 14 (2008), pp. 764-792.

[26] D. L. Donoho, Compressed sensing, IEEE Trans. Inform. Theory, 52 (2006), pp. 1289-1306.

$[27]$ D. L. Donoho, A. Maleki, I. Rahman, M. Shahram, and V. Stodden, Reproducible research in computational harmonic analysis, Computing in Science and Engineering, 11 (2009), pp. 8-18.

[28] D. L. Donoho And Y. Tsaig, Fast solution of $\ell_{1}$ minimization problems when the solution may be sparse, IEEE Trans. Inform. Theory, 54 (2008), pp. 4789-4812.

[29] D. L. Donoho, Y. Tsaig, I. Drori, and J.-L. Starck, Sparse solution of underdetermined linear equations by stagewise orthogonal matching pursuit, IEEE Trans. Inform. Theory, submitted.

[30] M. Elad, B. Matalon, And M. Zibulevsky, Coordinate and subspace optimization methods for linear least squares with non-quadratic regularization, Appl. Comput. Harmon. Anal., 23 (2007), pp. 346367.

[31] M. Elad, P. Milanfar, and R. Rubinstein, Analysis versus synthesis in signal priors, Inverse Problems, 23 (2007), pp. 947-968.

[32] M. A. T. Figueiredo AND R. D. NowAK, An EM algorithm for wavelet-based image restoration, IEEE Trans. Image Process., 12 (2003), pp. 906-916.

[33] M. A. T. Figueiredo, R. D. Nowak, And S. J. Wright, Gradient projection for sparse reconstruction: Application to compressed sensing and other inverse problems, IEEE J. Sel. Top. Signal Process., 1 (2007), pp. 586-597.

[34] J. Friedman, T. Hastie, and R. Tibshirani, Regularization paths for generalized linear models via coordinate descent, J. Stat. Softw., 33 (2010), pp. 1-22.

[35] T. Goldstein And S. Osher, The split Bregman method for L1 regularized problems, SIAM J. Imaging Sci., 2 (2009), pp. 323-343.

[36] E. T. Hale, W. Yin, and Y. Zhang, A Fixed-Point Continuation Method for 11-Regularized Minimization with Applications to Compressed Sensing, CAAM Technical Report TR07-07, Rice University, Houston, TX, 2007.

[37] E. T. Hale, W. Yin, And Y. Zhang, Fixed-point continuation for $\ell_{1}$-minimization: Methodology and convergence, SIAM J. Optim., 19 (2008), pp. 1107-1130.

[38] S. J. Kim, K. Koh, M. Lustig, S. Boyd, And D. Gorinevsky, An interior-point method for large-scale l1-regularized least squares, IEEE J. Sel. Top. Signal Process., 1 (2007), pp. 606-617.

[39] T. Lin and F. J. Herrmann, Compressed wavefield extrapolation, Geophysics, 72 (2007), pp. SM77SM93.

[40] I. LORIS, On the performance of algorithms for the minimization of $\ell_{1}$-penalized functionals, Inverse Problems, 25 (2009), 035008.

[41] I. Loris, M. Bertero, C. De Mol, R. Zanella, and L. Zanni, Accelerating gradient projection methods for $\ell_{1}$-constrained signal recovery by steplength selection, Appl. Comput. Harmon. Anal., 27 (2009), pp. 247-254.

[42] M. Lustig, D. L. Donoho, and J. M. Pauly, Sparse MRI: The application of compressed sensing for rapid MR imaging, Magn. Reson. Med., 58 (2007), pp. 1182-1195.

[43] S. Mallat, A Wavelet Tour of Signal Processing, Academic Press, San Diego, 1998.

[44] D. Needell And J. A. Tropp, CoSaMP: Iterative signal recovery from incomplete and inaccurate samples, Appl. Comput. Harmon. Anal., 26 (2009), pp. 301-321.

[45] Y. Nesterov, A method for solving the convex programming problem with convergence rate $\mathcal{O}\left(1 / k^{2}\right)$, Dokl. Akad. Nauk SSSR, 269 (1983), pp. 543-547 (in Russian); Soviet Math. Dokl., 27 (1983), pp. 372-376 (in English).

[46] Y. Nesterov, Introductory Lectures on Convex Optimization. A Basic Course, Kluwer Academic Publishers, Boston, 2004.

Copyright (C) by SIAM. Unauthorized reproduction of this article is prohibited. 
[47] Y. Nesterov, Smooth minimization of non-smooth functions, Math. Program. Ser. A, 103 (2005), pp. $127-152$

[48] Y. Nesterov, Gradient Methods for Minimizing Composite Objective Function, Technical report 2007/76, CORE, Université catholique de Louvain, Louvain-la-Neuve, Belgium, 2007.

[49] M. R. Osborne, B. Presnell, and B. A. Turlach, A new approach to variable selection in least squares problems, IMA J. Numer. Anal., 20 (2000), pp. 389-403.

[50] S. Osher, Y. MaO, B. Dong, And W. Yin, Fast linearized Bregman iteration for compressive sensing and sparse denoising, Commun. Math. Sci., 8 (2010), pp. 93-111.

[51] R. T. Rockafellar, Convex Analysis, Princeton Math. Ser. 28, Princeton University Press, Princeton, NJ, 1970.

[52] M. A. Saunders And B. Kim, PDCO: Primal-Dual Interior Method for Convex Objectives, Technical report, Stanford University, Stanford, CA, 2002; available online from http://www.stanford.edu/ group/SOL/software/pdco.html.

[53] R. Tibshirani, Regression shrinkage and selection via the lasso, J. Roy. Statist. Soc. Ser. B, 58 (1996), pp. $267-288$.

[54] P. Tseng, On Accelerated Proximal Gradient Methods for Convex-Concave Optimization, Technical report, Department of Mathematics, University of Washington, Seattle, WA; available online from http://www.math.washington.edu/ tseng/papers.html.

[55] E. van den Berg And M. P. Friedlander, Probing the Pareto frontier for basis pursuit solutions, SIAM J. Sci. Comput., 31 (2008), pp. 890-912.

[56] P. Weiss, L. Blanc-FÉRAud, AND G. Aubert, Efficient schemes for total variation minimization under constraints in image processing, SIAM J. Sci. Comput., 31 (2009), pp. 2047-2080.

[57] Z. Wen, W. Yin, D. Goldfarb, And Y. Zhang, A fast algorithm for sparse reconstruction based on shrinkage, subspace optimization, and continuation, SIAM J. Sci. Comput., 32 (2010), pp. 1832-1857.

[58] S. J. Wright, R. D. Nowak, and M. A. T. Figueiredo, Sparse reconstruction by separable approximation, IEEE Trans. Signal Process., 57 (2009), pp. 2479-2493.

[59] J. YANG, Y. Zhang, AND W. YIN, A fast alternating direction method for $T V \ell_{1}-\ell_{2}$ signal reconstruction from partial Fourier data, IEEE J. Sel. Top. Signal Process., 4 (2010), pp. 288-297.

[60] W. Yin, S. Osher, D. Goldfarb, And J. DARbon, Bregman iterative algorithms for $\ell_{1}$ minimization with applications to compressed sensing, SIAM J. Imaging Sci., 1 (2008), pp. 143-168.

[61] M. Zhu And T. Chan, An Efficient Primal-Dual Hybrid Gradient Algorithm for Total Variation Image Restoration, UCLA CAM Report [08-34], UCLA, Los Angeles, CA, 2008; available online from ftp:// ftp.math.ucla.edu/pub/camreport/cam08-34.pdf.

Copyright ( $\odot$ by SIAM. Unauthorized reproduction of this article is prohibited. 